\title{
Optimal synthesis of $\mathrm{p}$-xylene separation processes based on crystallization technology
}

\author{
Ricardo M. Lima, Ignacio E. Grossmann* \\ Department of Chemical Engineering, Carnegie Mellon University \\ Pittsburgh, PA 15213, United States
}

7th August 2008

\begin{abstract}
This paper addresses the synthesis and optimization of crystallization processes for $\mathrm{p}$ xylene recovery for systems with feed streams of high concentration, a case that arises in hybrid designs where the first step is commonly performed by adsorption. A novel superstructure and its corresponding mixed-integer nonlinear programming (MINLP) model are proposed. The distinct feature of this superstructure is the capability to generate optimum or near optimum flowsheets for a wide range of specifications of $\mathrm{p}$-xylene compositions in the feed stream of the process. In order to cope with the complexity of the MINLP model a two-level decomposition approach, consisting of the solution of an aggregated model and a detailed model, is proposed. The results obtained show good performance of the decomposition strategy, and the optimal flowsheets and p-xylene recoveries are in agreement with results reported in patents.
\end{abstract}

*Corresponding author. Tel.: +1 412268 2230; fax: +1 4122687139.

E-mail address: grossmann@cmu.edu 


\section{Introduction}

The separation of high purity p-xylene from a mixture of mixed xylenes -m-xylene, o-xylene, p-xylene and ethylbenzene- is industrially performed employing one of the following methods: (1) crystallization, (2) adsorption, or (3) a hybrid crystallization/adsorption process. ${ }^{1}$ Distillation is not a competitive technology due to the boiling point difference of only $2^{\circ} \mathrm{C}$ between $\mathrm{p}$-xylene and ethylbenzene (see Table 1) resulting in columns with high reflux ratios and a large number of trays.

\section{Table 1}

Adsorption is a competitive technology compared to crystallization. In adsorption the separation is achieved by exploiting the differences in affinity of the adsorbent for $\mathrm{p}$-xylene relative to the other components. The adsorbent is fixed in one column with multiple inlet and outlet ports, whereas the feed and the desorbent are swapped between ports of the simulating moving bed (SMB). Typical values for recovery per pass and purity of p-xylene are approximately $95.0 \mathrm{wt} \%$ and $99.7 \mathrm{wt} \%{ }^{1,2}$

Crystallization based processes exploit the large freezing point difference between p-xylene and the remaining components in the mixture. Typical processes consist of one or two crystallization stages operating at different temperature levels, liquid/solid separation devices using different types of centrifuges (imposed by operation ranges of feed solid concentrations and of feed temperature), melting stages with slurry drums and heat exchangers, and a final stage of purification involving centrifuges with wash streams. The units are organized by stages in series and/or parallel configurations giving rise to complex structures. A simple flowsheet that illustrates crystallization separation based processes without heat integration is shown in Figure 1.

\section{Figure 1}

In the specific case of the $\mathrm{p}$-xylene separation, the maximum recovery of $\mathrm{p}$-xylene is limited by the eutectic point, i.e. the temperature level where a second component starts to crystallize. Typical values for recovery are between $60-65 \mathrm{wt} \%$ for feed streams with about $20 \mathrm{wt} \%$ of p-xylene. ${ }^{1,3}$ 
This limitation is one of the main drawbacks of crystallization when processing feeds with a low concentration of p-xylene. For higher concentrations the recovery rates can be above $90 \mathrm{wt} \%$.

Several commercial processes have been developed by industry, namely the Amoco, Chevron, Arco or Philips ${ }^{1}$ processes. These differ in the type of crystallizers employed, topology and process conditions. The design of crystallization processes has been the subject of several US patents in the last decades. The first patents proposed crystallization processes to recover p-xylene from a mixture with $20 \mathrm{wt} \%$ p-xylene. ${ }^{4-8}$ The more recent trend has been to design hybrid processes involving a first stage based on adsorption and a second stage based on crystallization. ${ }^{3,9-11}$ This is because it can be more economical to separate the p-xylene from mixtures with approximately 20 wt \% p-xylene with adsorption due to the high recovery rate obtained per pass when compared with crystallization. ${ }^{3}$ The aforementioned works aim to design energy efficient processes by minimizing the energy consumption through the minimization of the number of melting stages and refrigeration loads that are required. To accomplish this objective, these works have suggested different operating conditions and different process configurations. For example Hubbell and Rutten $^{3}$ proposed six alternative flowsheets in order to deal with feed streams with different $\mathrm{p}$-xylene compositions, while Eccli and Fremuth ${ }^{9}$ explore the fact that for fixed feed streams with high concentration of $\mathrm{p}$-xylene, decreasing the operating temperature of crystallizers the $\mathrm{p}$-xylene recovery rate increases. However, a systematic approach for the synthesis of p-xylene separation processes is lacking.

In general, the synthesis of flowsheets can be addressed using one of the following approaches ${ }^{12}$ : 1) hierarchical decomposition, or 2) mathematical programming. $\mathrm{Ng}$ and co-workers ${ }^{13-17}$ have extensively studied the application of hierarchical approaches based on step-by-step procedures using rules and phase diagrams, as tools for the synthesis of crystallization processes. These authors have studied the synthesis of fractional crystallization processes, crystallization systems with multiple stages, and the necessary systems around crystallizers. A thorough discussion about the hierarchical approach can be found elsewhere. ${ }^{15,18}$ In addition, Chang and $\mathrm{Ng}^{17}$ have also recognized the value of the mathematical programming approach, and proposed a conceptual superstructure of a 
crystallization system.

The synthesis and optimization of crystallization processes employing mathematical programming techniques is an area where little work has been reported. The studies tend to be dominated by works related with reactor networks, distillation, heat exchanger networks, mass exchange networks and utility systems. ${ }^{19}$

Cisternas et al. ${ }^{20}$ were the first to devise a flowsheet synthesis methodology based on mathematical programming for the design of crystallization processes. These authors developed a network comprising four subnetworks: (1) thermodynamic states, (2) tasks, (3) heat integration, and (4) a filtration and cake washing subnetwork. In their network of thermodynamic states the solidliquid equilibrium conditions are represented by saturation conditions at specific temperatures. They proposed models for crystallization and separation devices for several salt separation processes, which result in Mixed-Integer Linear Programming (MILP) models.

Méndez et al. ${ }^{21}$ developed an MINLP model for the synthesis of p-xylene recovery from a stream with approximately $20 \mathrm{wt} \%$ of p-xylene. These authors proposed a new superstructure for the crystallization stages, and furthermore considered the optimization of several process configurations individually. The major decisions involved the topological configuration and operating conditions. In all process configurations they considered the same superstructure for the crystallizers, but in each one they considered different stages, e.g. number of melting stages, number of crystallization stages and separation stages.

The aim of this work is to develop a mathematical programming approach for the optimal synthesis of p-xylene separation processes based on crystallization. We propose a novel superstructure, an MINLP model, and a decomposition strategy to cope with the complexity of the model. In addition, in this study we will only consider feed streams with compositions of p-xylene greater than 65 wt $\%$. This situation occurs in the design of crystallization as a second stage of hybrid processes, where the feed stream to the crystallization process has a high concentration of p-xylene. 


\section{Problem statement}

Given is a multicomponent feed with fixed compositions of mixed xylenes and a production target of p-xylene in terms of product quantity and quality. The problem then consists in synthesize a flowsheet consisting of crystallizers, centrifuges, slurry drums, splitters, mixers, and heat exchangers, in which the number of these process units must be determined as well as their operating conditions. The goal is to minimize the total annualized cost of the process.

\section{Superstructure}

In order to address the proposed synthesis problem a superstructure was developed, and then modeled as an MINLP problem. Figure 2 illustrates the compact version of the proposed superstructure that has embedded flowsheet structures to be analyzed.

\section{Figure 2}

The superstructure used in this work is delimited in Figure 2 by a discontinuous line, where the feed stream is the output of a p-xylene enriching zone that could be a low temperature crystallization, a selective adsorption or a toluene disproportionation process. ${ }^{10}$

This superstructure includes all the flowsheets studied by Méndez et al. ${ }^{21}$, but with modifications in the centrifuges blocks and with many new connections between stages. The blocks shown in Figure 2 correspond to superstructures of subsystems, i.e. CSI and CSII represent two superstructures of crystallizers, the blocks CFI, CFII, and CFIII stand for structures of centrifuges with different characteristics, and the blocks SLDI and SLDII represent structures involving slurry drums.

Figure 3 shows the two superstructures associated with blocks CSI and CSII. In the first crystallization stage the lower bound for the outlet temperature of crystallizers is the eutectic temperature, while in the second a higher bound on the outlet temperature is imposed so that the superstructure can consider two stages of crystallization at different temperatures. The block CSII involves less 
flexibility in terms of interconnections between units because it is expected to treat streams with high concentrations of p-xylene and at higher temperatures. The superstructure in Figure 3, when compared with the one proposed by Méndez et al. ${ }^{21}$, includes more feed streams and new streams from the splitter after each crystallizer to the block CFIII.

\section{Figure 3}

The latter streams increase the flexibility of the superstructure to generate efficient flowsheets for high compositions of $\mathrm{p}$-xylene in the feed stream of the process. This means that for a process feed stream with 90 wt \% of p-xylene, the output from crystallizers does not need to go through the block of centrifuges CFI, but it can be sent directly to the block with centrifuges CFIII. The blocks CFI, CFII, and CFIII are shown in Figure 4.

\section{Figure 4}

The first block includes a set of centrifuges in parallel operating at the same temperature since they have a single feed. CFII and CFIII have the flexibility of having centrifuges in parallel working at different temperatures since they handle multiple feeds. These three blocks represent three different types of centrifuges, with different ranges of operation in terms of inlet total flowrate, inlet solid p-xylene flowrate and minimum inlet temperature. Therefore, the three blocks are not alternatives between them. In addition, based on the purity of the cake obtained, the final product is set to be the output cake from the centrifuges CFIII

The structures merged in blocks SLDI and SLDII, which involve different interconnections, are represented in Figure 5.

\section{Figure 5}

The slurry drums are used to increase the temperature of some streams in order to meet the temperature constraints imposed by the centrifuges. From the practical point of view the rise of temperature in slurry drums leads to the melt of crystals contributing also for the elimination of impurities in the crystals. However, this phenomenon is not considered in our model. 


\section{Mixed-integer nonlinear programming (MINLP) model}

The MINLP model used in this work is based on the model proposed by Méndez et al. ${ }^{21}$ The three key ideas of their model are (1) the inclusion of a crystallizer network in the first crystallization stage, (2) the way the components are defined in the model, and (3) the submodel used for dealing with the solubility prediction.

The main feature of the crystallization network proposed by those authors is the imposition of an order between them, with recycles only to downstream crystallizers. Here, the network is augmented not only due to the existence of more external recycle streams, but also because of the streams that link this network with the block CFIII (see Figure 3).

Méndez et al. ${ }^{21}$ proposed a model using individual flowrate components instead of concentrations, and considered 3 components: (1) solid p-xylene, (2) liquid p-xylene, and (3) m-xylene, o-xylene and ethylbenzene aggregated as one component. Therefore, the liquid phase is made of liquid p-xylene and the aggregated component.

Regarding the solubility predictions, these authors applied a correction in some mass balances based on the use of a smooth approximation of the max function ${ }^{22}$ to correct the eventual surplus predicted by the nonlinear solubility correlation. However, in this work, the small value used in the smooth approximation revealed to have an impact in the final results. Therefore, the smooth approximation is substituted by an approach that makes use of $0-1$ variables. In this model the level of detail involves the existence of two phases with the corresponding $\mathrm{p}$-xylene equilibrium between liquid and solid phases, associated with the previously mentioned correction. However, it does not involve prediction of particle size or crystal impurities, which is a critical factor for efficient liquid/solid separation where larger crystals are favored. ${ }^{1}$ A greatly rigorous model involving crystal size distribution and the phenomena that affect it would increase the model complexity making the problem extremely difficult to solve. Nevertheless, the models of the centrifuges involve mass balances with nonlinearities and several parameters that predict cake and filtrate streams conditions, while in slurry drums the volume size involves density calculations and it is assumed a specific residence time. Therefore, with the current level of detail associated with the described superstructure 
our model offers the possibility to explore alternative flowsheets and operating conditions.

The proposed model, which is described in the next subsections, includes several nonlinearities, mainly in individual component mass balances for the splitters and heat balances, as well as discontinuities in the solubility correlations, giving rise to a nonconvex MINLP problem of large dimension.

\section{Objective function}

The objective function, given by Equation 1, represents the total annualized cost that is to be minimized considering both operating and capital investments. The operating costs include feed, rejected filtrate and energy costs (electricity, refrigeration and steam), while the capital investments include all the costs related with the crystallizers, the different type of centrifuges used, heat exchangers, drums and the rejected filtrate stream:

$$
\begin{aligned}
Z= & C_{F D} F D-C_{R} F_{s^{R}}+C_{E} \sum_{u \in U^{C R T}} \sum_{s \in S_{u}^{O}} \Theta\left(T_{s}, H R_{s}\right)+C_{R} \sum_{u \in U^{C R T}} \sum_{s \in S_{u}^{O}} H R_{s}^{\nu_{u}}+ \\
& C_{S} \sum_{u \in U^{H E H}} \sum_{s \in S_{u}^{O}} H A_{s}+\sum_{u \in U^{C F I}}\left(\alpha_{u} y_{u}+C_{E} E L E_{u}\right)+\sum_{u \in U^{C F I I}}\left(\alpha_{u} y_{u}+C_{E} E L E_{u}\right)+ \\
& \sum_{u \in U^{C F I I I}}\left(\alpha_{u} y_{u}+C_{E} E L E_{u}\right)+\sum_{u \in U^{C R T}} \beta_{u} D_{u}^{\nu_{u}}+\sum_{u \in U^{S L D}} \beta_{u} D_{u}^{\nu_{u}}+\sum_{u \in U^{H E H}} \beta_{u} D_{u}^{\nu_{u}}+ \\
& \sum_{u \in U^{M X R}} \beta_{u} D_{u}^{\nu_{u}}+P X_{O F F S E T}\left(\lambda_{F L} C_{F L}+\lambda_{E} C_{E}+\lambda_{F D} C_{F D}\right)+C_{H E} \Omega\left(F_{s}\right)
\end{aligned}
$$

where $C_{S}, C_{F D}, C_{R}, C_{F L}, C_{E}, C_{H E}, \alpha_{u}, \beta_{u}, \lambda_{F L}, \lambda_{E}, \lambda_{F D}$, and $\nu_{u}$ are cost parameters, $F D$ is the flowrate of the process feed, $F_{s^{R}}$ is the flowrate of the rejected filtrate, $T_{s}$ is the temperature of stream $s, H R_{s}$ is the heat removed in crystallizers from stream $s, H A_{s}$ is the heat added in heat exchangers to stream $s, y_{u}$ is a discrete variable associated with the process unit $u, E L E_{u}$ is the electricity required by centrifuges and the refrigeration system, $D_{u}$ denotes the size of unit $u, P X_{O F F S E T}$ is the wt $\%$ of p-xylene in the rejected filtrate stream, and $\Theta$ and $\Omega$ are nonlinear functions. The units considered for the objective function are monetary units per year (m.u./year). 


\section{Mass balances}

The individual component mass balances for all units are divided into two equations. The first, Equation 2, is the mass balance for the components in the liquid phase not considering the liquid p-xylene:

$$
\sum_{s \in S_{u}^{I}} F_{s L}=\sum_{s \in S_{u}^{O}} F_{s L} \quad \forall u \in U
$$

while the second equation takes into account the phase change of p-xylene, and therefore it is applied to solid and liquid p-xylene:

$$
\sum_{s \in S_{u}^{I}}\left(F_{s L X}+F_{s S X}\right)=\sum_{s \in S_{u}^{O}}\left(F_{s L X}+F_{s S X}\right) \quad \forall u \in U
$$

where $F_{s L}$ is the individual flowrate of the aggregated liquid component, $F_{s L X}$ is the individual flowrate of p-xylene in the liquid phase in the stream $s$ and $F_{s S X}$ is the individual flowrate of pxylene in the solid phase in the stream $s$. The flowrate of each stream is given by the summation of the individual flowrate components, $F_{s c}$,

$$
F_{s}=\sum_{c \in C} F_{s c} \quad \forall s \in S
$$

Whenever the feed composition is known the following equation is considered:

$$
F D_{c}=F D \zeta_{c} \quad \forall c \in C
$$

where $\zeta_{c}$ and $F D_{c}$ are the wt $\%$ and individual flowrate, respectively, of each component in the feed stream to the process. The flowrate of the liquid phase, $F L_{s}$, is given by,

$$
F L_{s}=F_{s L X}+F_{s L} \quad \forall s \in S
$$


The following equation is only used for splitters, where a split fraction is used to set the individual output flowrate for each component.

$$
F_{s c}=\xi_{u s} F_{s^{\prime} c} \quad \forall u \in U^{S P L}, \forall c \in C, \forall s^{\prime} \in S_{u}^{I}, \forall s \in S_{u}^{O}
$$

\section{Heat balances}

$$
\begin{aligned}
& \sum_{c \in C} F_{s c} \Lambda_{c}\left(T_{s}\right)=\sum_{c \in C} F_{s^{\prime} c} \Lambda_{c}\left(T_{s^{\prime}}\right)+H R_{s^{\prime}} \quad \forall u \in U^{C R T}, \forall s \in S_{u}^{I}, \forall s^{\prime} \in S_{u}^{O} \\
& \sum_{s \in S_{u}^{I}} \sum_{c \in C} F_{s c} \Lambda_{c}\left(T_{s}\right)=\sum_{c \in C} F_{s^{\prime} c} \Lambda_{c}\left(T_{s^{\prime}}\right) \quad \forall u \in U^{S L D} \cup U^{M X R}, \forall s^{\prime} \in S_{u}^{O} \\
& \sum_{c \in C} F_{s c} \Lambda_{c}\left(T_{s}\right)=\sum_{c \in C} F_{s^{\prime} c} \Lambda_{c}\left(T_{s^{\prime}}\right)-H A_{s^{\prime}} \quad \forall u \in U^{H E H}, \forall s \in S_{u}^{I}, \forall s^{\prime} \in S_{u}^{O} \\
& T_{s}=T_{s^{\prime}} \quad \forall u \in U^{S P L}, \forall s \in S_{u}^{I}, \forall s^{\prime} \in S_{u}^{O} \\
& H T C_{u}=\Phi\left(H R_{s}\right) \quad \forall u \in U^{C R T}, \forall s \in S_{u}^{O} \\
& H R_{s}=H T C_{u} D_{u}\left[\frac{\Delta T_{1} \Delta T_{2}\left(\Delta T_{1}+\Delta T_{2}\right)}{2}\right]^{1 / 3} \quad \forall u \in U^{C R T}, \forall s \in S_{u}^{O} \\
& H A_{s}=H C U_{u} D_{u}\left[\frac{\Delta T_{1} \Delta T_{2}\left(\Delta T_{1}+\Delta T_{2}\right)}{2}\right]^{1 / 3} \quad \forall u \in U^{H E H}, \forall s \in S_{u}^{O}
\end{aligned}
$$

where $\Lambda_{c}$ are nonlinear functions of $T_{s}$ used to calculate the heat capacity of component $c, H T C_{u}$ is the heat transfer coefficient for crystallizers, $\Phi$ is a nonlinear function to calculate $H T C_{u}$. In Equations 13 and $14 D_{u}$ are the surface areas of the crystallizers and heat exchangers, respectively, and $H C U_{u}$ the heat transfer coefficients for heat exchangers. 


\section{Solubility}

The solubility of $\mathrm{p}$-xylene, $\sigma_{s}$, is defined by the following expression:

$$
\sigma_{s}=\Gamma\left(T_{s}\right) \quad \forall s \in S
$$

where $\Gamma$ is a nonlinear function, with the general form of the functions illustrated in Figure 6.

\section{Figure 6}

In each stream a two-component system (p-xylene - aggregated component) is considered, where the p-xylene can exist in the solid state and in the liquid solution. Based on the p-xylene solubility behaviour, two cases should be considered: 1) the solubility is greater than the concentration of p-xylene in the liquid, and 2) the solubility is less or equal than the concentration of p-xylene in the liquid. The first case may occur for some streams, e.g. for the rejected filtrate of the centrifuges from block CFI, where the amount of solid p-xylene may be equal to zero. In this case $\sigma_{s}>F_{s L X} / F L_{s}$, and therefore, to correct an eventual surplus predicted by the nonlinear solubility correlation, Méndez et al. ${ }^{21}$ applied the following solubility correction:

$$
\begin{gathered}
F_{s L X}=\sigma_{s} F L_{s}-\max \left\{0, \varepsilon_{s}\right\} \quad \forall u \in\left(U^{M X R 2} \cup U^{H E H}\right), \forall s \in S_{u}^{O} \\
F_{s L X}=\sigma_{s} F L_{s}-\max \left\{0, \varepsilon_{s}\right\} \quad \forall u \in\left(U^{C F I} \cup U^{C F I I I}\right), \forall s \in\left(S_{C F I}^{R F} \cup S_{C F I I I}^{S F} \cup S_{C F I I I}^{R F}\right)
\end{gathered}
$$

in addition with the following equations:

$$
\begin{gathered}
\varepsilon_{s}=\sigma_{s} F L_{s}-F_{s L X}-F_{s S X} \quad \forall u \in\left(U^{M X R 2} \cup U^{H E H}\right), \forall s \in S_{u}^{O} \\
\varepsilon_{s}=\sigma_{s} F L_{s}-F_{s L X}-F_{s S X} \quad \forall u \in\left(U^{C F I} \cup U^{C F I I I}\right), \forall s \in\left(S_{C F I}^{R F} \cup S_{C F I I I}^{S F} \cup S_{C F I I I}^{R F}\right)
\end{gathered}
$$

where $\varepsilon_{s}$ is the amount of $\mathrm{p}$-xylene predicted in excess. From the above equations, for the first case $F_{s L X}=\sigma_{s} F L_{s}-\varepsilon_{s}$, and $F_{s S X}=0$. In the second case the streams contain solid p-xylene, and the above equations become $F_{s L X}=\sigma_{s} F L_{s}$, and $F_{s S X}=-\varepsilon_{s}$ with $\varepsilon_{s}<0$. 
The correction proposed by Méndez et al. ${ }^{21}$ was based on the definition of a smooth approximation $^{22}$ to represent the max function in the equations. In this work the max function is replaced by a mixed-integer formulation using the convex hull reformulation ${ }^{23}$ as expressed by Equation 20 where $\varepsilon_{s}^{L}<0$ and $\varepsilon_{s}^{U}>0$. Thus, the term $\phi=\max \left\{0, \varepsilon_{s}\right\}$ is formulated as:

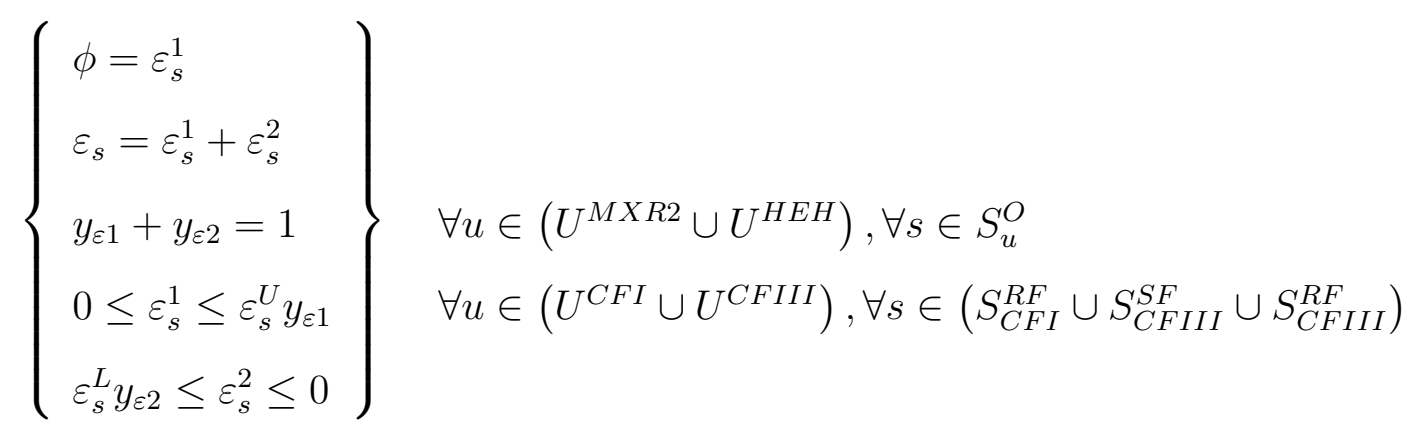

In order to avoid the crystallization of a second component, Equation 21 ensures that the temperature of all streams is above the eutectic point, $T^{e u t}=205.7 \mathrm{~K}$ and a p-xylene concentration of 7.4 wt \%. Equation 22 sets an upper bound on the temperature of the output streams from crystallizers in order to guarantee specific operating conditions.

$$
\begin{array}{ll}
T_{s} \geq T^{e u t} & \forall s \in S \\
T_{s} \leq T^{i c t} & \forall u \in U^{C S I}, \forall s \in S_{u}^{O}
\end{array}
$$

\section{Density correlations}

The volumes of some drums are calculated using density correlations:

$$
\begin{aligned}
& \rho_{s}=\psi\left(T_{s}\right) \quad \forall u \in U^{M X R}, \forall s \in S_{u}^{O} \\
& \rho_{s}=\psi\left(T_{s}\right) \quad \forall u \in U^{S L D}, \forall s \in S_{u}^{O}
\end{aligned}
$$

where $\psi$ is a nonlinear function and $\rho_{s}$ the density of the stream $s$. 


\section{Centrifuges}

The separation performance of each type of centrifuge is defined by a set of parameters $\kappa_{u}$ that are associated with Equation 25 to determine the flowrates and compositions of each outlet stream -cake, rejected and screen filtrate- from the centrifuges. Equation 25 involves a set of linear equations and nonlinear equations,

$$
\begin{aligned}
& F_{s^{\prime} c}=\Phi\left(F_{s c}, \kappa_{u}\right) \quad \forall u \in\left(U^{C F I} \cup U^{C F I I} \cup U^{C F I I I}\right), \forall s \in S_{u}^{I}, \forall s^{\prime} \in S_{u}^{O} \\
& T_{s^{\prime}}=\Phi\left(T_{s}, \kappa_{u}\right) \quad \forall u\left(U^{C F I} \cup U^{C F I I} \cup U^{C F I I I}\right), \forall s \in S_{u}^{I}, \forall s^{\prime} \in S_{u}^{O} \\
& E L E_{u}=\Phi\left(F_{s S X}, F_{s}, y_{u}\right) \quad \forall s \in S_{u}^{I}, \forall u \in U^{C F I} \\
& E L E_{u}=\Phi\left(F_{s}, y_{u}\right) \quad \forall s \in S_{u}^{I}, \forall u \in U^{C F I I} \\
& E L E_{u}=\Phi\left(F_{s S X}, F_{s}, y_{u}\right) \quad \forall s \in S_{u}^{I}, \forall u \in U^{C F I I I}
\end{aligned}
$$

where $E L E_{u}$ is the electricity required for each centrifuge, which is a function of the inlet flowrate (total and/or solid p-xylene). In the optimum flowsheets, the centrifuges are assumed to be located in specific locations of the process according to their operating conditions. These are related to feed flowrate to the centrifuge, viz. maximum concentration of solids or maximum flowrate, and

minimum temperature. These constraints are represented by Equations 30 to 33. Equation 30 defines a constraint on the maximum value of solid p-xylene flowrate, $F_{s S X}^{U}$, in the centrifuges feed, while equation 31 defines the maximum feed flowrate, $F_{s}^{U}$, for the centrifuges in the block CFIII.

$$
\begin{aligned}
& F_{s S X} \leq F_{s S X}^{U} y_{u} \quad \forall u \in\left(U^{C F I} \cup U^{C F I I}\right), \forall s \in S_{u}^{I} \\
& F_{s c} \leq F_{s}^{U} y_{u} \quad \forall u \in U^{C F I I I}, \forall s \in S_{u}^{I} \\
& \sum_{s \in S_{u}^{O}} F_{s c} \leq F_{s}^{U} y_{u} \quad \forall u \in U
\end{aligned}
$$




$$
T_{s} \geq T_{s}^{L} \quad \forall u \in\left(U^{C F I I} \cup U^{C F I I I}\right), \forall s \in S_{u}^{I}
$$

Equation 33 imposes a lower bound on the inlet temperatures of the centrifuges to keep the streams viscosity and density in ranges so they can operate effectively. ${ }^{1}$ In addition, in the last stage a lower bound on the inlet temperatures also prevents the washing liquid to crystallize, which would lead to a reduction of the efficiency of the washing procedure.

\section{Production targets}

The desired amount and quality of the p-xylene product are set by the following two equations:

$$
\begin{aligned}
& \sum_{c \in C} F_{s^{P} c} \geq F^{\text {min }} \\
& F_{s^{P} L X}+F_{s^{P} S X} \geq \eta \sum_{c \in C} F_{s^{P} c}
\end{aligned}
$$

where $F^{\text {min }}$ denotes the required output flowrate and $\eta$ the minimum p-xylene purity in the output stream. The minimum number of units in the block CFIII can be set as the inequality,

$$
\sum_{u \in U^{C F I I I}} y_{u} \geq \frac{F_{s^{P} c}}{F_{s S X}^{U}}
$$

since the minimum amount of p-xylene in the output stream is known.

\section{Process units additional constraints}

Some of the remaining units have specific additional mass balances equations. The next two equations set the wt $\%$ of solid p-xylene in the output stream as a function of a parameter $\kappa_{u}$, for the slurry drums and for the crystallizers of the second stage,

$$
\sum_{s \in S_{u}^{O}}\left(F_{s S X}-F_{s} \kappa_{u}\right)=0 \quad \forall u \in U^{S L D}
$$




$$
\begin{array}{ll}
\sum_{s \in S_{u}^{O}} F_{s S X}-\kappa_{u} F_{s}=0 & \forall u \in U^{C S I I} \\
\sum_{s \in S_{u}^{I}} F_{s S X} \geq \sum_{s \in S_{u}^{O}} F_{s S X} & \forall u \in U^{H E H}
\end{array}
$$

The above equation forces the inlet flowrate of the solid p-xylene to the heat exchangers to be greater or equal than the output flowrate of the solid p-xylene. The wt $\%$ of p-xylene in the rejected filtrate stream is defined by:

$$
\frac{F_{s^{R} L X}+F_{s^{R} S X}}{F_{s^{R}}} \leq P X_{O F F S E T}
$$

where $F_{s^{R} L X}$ is the individual flowrate of liquid p-xylene, $F_{S^{R} S X}$ is the individual flowrate of solid p-xylene, and $F_{s^{R}}$ is the flowrate of the rejected filtrate stream. The temperature of the rejected filtrate is always above the eutectic temperature because of the temperature increase in the centrifuges, (defined by Equation 26), leading also to a slightly increase of the concentration of pxylene in the liquid. In addition, the amount of solid p-xylene in the rejected filtrate is considerably less than the amount of solid p-xylene in any stream at the eutectic conditions. This is important because there is a cost associated with the concentration of $\mathrm{p}$-xylene in the rejected filtrate stream that is recycled.

\section{Logic constraints}

When using integer cuts, solutions like $\left\{s \in S_{u}^{O}, u \in U^{C R T}: y_{u}=1, F_{s}=0\right\}$ can occur (i.e. a crystallization unit is selected but with zero flow), and consequently the objective function value would not correspond to the topology of the process. Therefore, the following constraint was included:

$$
\sum_{s \in S_{u}^{O}} F_{s c} \geq F_{S}^{L} y_{u} \quad \forall u \in U^{C R T}
$$


where $F_{s}^{L}$ is the lower bound for the flowrate of stream $s$. One of the main features of the crystallization network is the order of selection of crystallizers, which is accomplished by the following equation:

$$
y_{u} \geq y_{u+1} \quad u=1, \ldots, i
$$

where $i$ is the maximum number of crystallizers in the block CSI.

The imposition of the constraint defined in Equation 38 requires the introduction of a bypass stream, (see Figure 7).

\section{Figure 7}

The goal of this stream is to deactivate those constraints associated with the second stage of crystallization when $F_{s}>0, D_{u}=0, s \in S_{u}^{O}, u \in U^{C S I I}$, i.e. when the flowrate is nonzero but the transfer area is zero, the constraint from Equation 38 cannot be met. Therefore, using the notation from Figure 7, this can be represented by a disjunction as follows:

$$
\left[\begin{array}{c}
\neg y_{u}, \forall u \in U^{C S I I} \\
F_{602} \geq 0 \\
F_{602 c} \geq 0, c \in C \\
F L_{602} \geq 0 \\
\xi_{602} \geq 0 \\
F_{603}=0 \\
F_{603 c}=0, c \in C \\
F L_{603}=0 \\
\xi_{603}=0
\end{array}\right] \vee\left[\begin{array}{c}
\bigvee_{u \in U^{C S I I}}\left[y_{u}\right] \\
F_{602}=0 \\
F_{602 c}=0, c \in C \\
F L_{602}=0 \\
\xi_{602}=0 \\
F_{603} \geq 0 \\
F_{603 c} \geq 0, c \in C \\
F L_{603} \geq 0 \\
\xi_{603} \geq 0
\end{array}\right]
$$

Introducing the binary variable $z$, where $z=1$ means that $\left\{\exists u \in U^{C S I I}: y_{u}=1\right\}$, this can be 
represented in logic form as:

$$
\bigvee_{u \in U^{C S I I}} y_{u} \Leftrightarrow z
$$

which is equivalent to:

$$
\left(\bigvee_{u \in U^{C S I I}} y_{u} \Rightarrow z\right) \wedge\left(z \Rightarrow \bigvee_{u \in U^{C S I I}} y_{u}\right)
$$

Transforming these logic propositions into inequalities yields ${ }^{24}$ :

$$
\begin{gathered}
z-y_{u} \geq 0 \quad \forall u \in U^{C S I I} \\
\sum_{u \in U^{C S I I}} y_{u}-z \geq 0
\end{gathered}
$$

Therefore, the above disjunction can be additionally represented by:

$$
\begin{aligned}
& F_{603} \leq F_{603}^{U} z \\
& F_{603 c} \leq F_{603 c}^{U} z \\
& F L_{603} \leq F L_{603}^{U} z \\
& \xi_{603} \leq \xi_{603}^{U} z \\
& F_{602} \leq F_{602}^{U}(1-z) \\
& F_{602 c} \leq F_{602 c}^{U}(1-z) \\
& F L_{602} \leq F L_{602}^{U}(1-z) \\
& \xi_{602} \leq \xi_{602}^{U}(1-z)
\end{aligned}
$$

Logic constraints are also used to choose only one output stream out of two streams from two 
specific splitters. These constraints are introduced to avoid splitting one stream into two streams, heat one of the streams and afterwards mix them again. Figure 8 illustrates an extract of the superstructure with the two splitters where the constraints were imposed.

\section{Figure 8}

Following the notation used in Figure 8, these constraints can be logically represented as:

$$
\left[\begin{array}{c}
z_{1} \\
F_{913} \geq 0 \\
F_{913 c} \geq 0 \\
F L_{913} \geq 0 \\
\xi_{913} \geq 0 \\
F_{914}=0 \\
F_{914 c}=0 \\
F L_{914}=0 \\
\xi_{914}=0
\end{array}\right] \vee\left[\begin{array}{c}
\neg z_{1} \\
F_{913}=0 \\
F_{913 c}=0 \\
F L_{913}=0 \\
\xi_{913}=0 \\
F_{914} \geq 0 \\
F_{914 c} \geq 0 \\
F L_{914} \geq 0 \\
z_{914} \geq 0 \\
F_{915} \geq 0 \\
F_{915 c} \geq 0 \\
F L_{915} \geq 0 \\
\xi_{915} \geq 0 \\
F_{916}=0 \\
F_{916 c}=0 \\
\xi_{916}=0
\end{array}\right] \vee\left[\begin{array}{c}
\neg z_{916} \\
F_{915}=0 \\
F_{915 c}=0 \\
F L_{915}=0 \\
\xi_{915}=0 \\
F_{916} \geq 0 \\
F_{916 c} \geq 0 \\
F L_{916} \geq 0 \\
\xi_{916} \geq 0
\end{array}\right]
$$


and converted into inequality constraints using a big-M transformation:

$$
\begin{aligned}
& F_{913} \leq F_{913}^{U} z_{1} \\
& F_{913 c} \leq F_{913 c}^{U} z_{1} \\
& F L_{913} \leq F L_{913}^{U} z_{1} \\
& \xi_{913} \leq \xi_{913}^{U} z_{1} \\
& F_{914} \leq F_{914}^{U}\left(1-z_{1}\right) \\
& F_{914 c} \leq F_{914 c}^{U}\left(1-z_{1}\right) \\
& F L_{914} \leq F L_{914}^{U}\left(1-z_{1}\right) \\
& \xi_{914} \leq \xi_{914}^{U}\left(1-z_{1}\right) \\
& F_{915} \leq F_{915}^{U} z_{2} \\
& F_{915 c} \leq F_{915 c}^{U} z_{2} \\
& F L_{915} \leq F L_{915}^{U} z_{2} \\
& \xi_{915} \leq \xi_{915}^{U} z_{2} \\
& F_{916} \leq F_{916}^{U}\left(1-z_{2}\right) \\
& F_{916 c} \leq F_{916 c}^{U}\left(1-z_{2}\right) \\
& F L_{916} \leq F L_{916}^{U}\left(1-z_{2}\right) \\
& \xi_{916} \leq \xi_{916}^{U}\left(1-z_{2}\right)
\end{aligned}
$$

where $z_{1}=1$ if $F_{913} \geq 0$, and $z_{2}=1$ if $F_{915} \geq 0$. 


\section{Solution approach}

The MINLP model described was implemented using the modeling system GAMS. ${ }^{25}$ The model has 3054 constraints, 2991 continuous variables and 101 binary variables. The latter are associated with the existence of equipment units, and with the solubility equations and logic constraints. The nonlinearities arise from the mass balances for the splitters, heat balances, density correlations, solubility correlations and from the mass balances for centrifuges, resulting in a nonconvex MINLP problem.

The first case that we have studied was the synthesis of p-xylene recovery from a stream with 65 wt $\%$ of p-xylene. As a first approach DICOPT ${ }^{26}$ was employed to solve the MINLP problem. However, there were difficulties for obtaining feasible solutions for the relaxed MINLP problem, resulting on several failures to get optimum solutions. Specifying a good starting point and upper bounds based on process insights allowed DICOPT to obtain optimum solutions for the relaxed MINLP problem and nonlinear programming (NLP) subproblems. However, because of the nonconvexities of the model this solver was highly dependent on the starting point of the integer variables, and several suboptimal solutions were obtained. As a second approach, the solver GAMS/SBB was employed with a maximum number of nodes set to 500, and then its output solution was used as an initial point for GAMS/DICOPT, but a clear improvement was not observed.

In order to obtain better solutions to the MINLP model in shorter time and in a more robust way, a two-level decomposition approach is proposed. This approach consists of the solution of an aggregated model and a detailed model. In the proposed superstructure (see Figure 2) the block CFI corresponds to a set of centrifuges in parallel as illustrated in Figure 9a).

\section{Figure 9}

This block and the additional blocks CFII and CFIII, suggested an aggregated model, where the set of units in parallel would be substituted by only one unit (see Figure 9b)).

The two key ideas in the aggregated model are: (1) merging the units in centrifuge blocks and slurry drums into single input-output blocks so that the aggregated model is defined in the space of 
interconnection of major blocks, and (2) the relaxation of the constraints that set an upper bound on the inlet flowrate of each centrifuge unit in order to meet the same production targets. Figures 9, 10 and 11 show the set of centrifuges in parallel in the detailed model and the respective structure defined in the aggregated model.

\section{Figure 10}

\section{Figure 11}

From these figures one can see the degree of simplicity achieved by aggregating each of the sets of centrifuges in parallel into only one centrifuge. This means that in the mathematical model a large number of equations and variables are replaced by equations of a single equivalent unit.

The two-level decomposition approach is applied accordingly with the algorithm illustrated in Figure 12.

\section{Figure 12}

Note that the aggregated and detailed models are formulated as MINLP models. The optimum solution of the aggregated model is used to initialize and define the superstructure of the detailed model. In particular, streams with zero flowrate in the solution of the aggregated model are removed from the superstructure of the detailed model. The aggregated model yields an upper bound on the total annual cost (see next section) while the detailed model yields a lower bound. The algorithm iterates between the solution of the aggregated and detailed model until the difference between the bounds is less than a specified tolerance. Between each iteration two integer cuts are added to expedite the search. The next three subsections give the details about the bounds of each problem, the integer cuts added between the two levels, and the definition and initialization of the detailed model.

\section{Bounds on the cost}

Generally for a minimization problem, it would be expected that the aggregated model yields a lower bound on the objective function due to relaxations on some constraints or the underestimation 
of the objective function. However, in this work the aggregated model yields an upper bound. This bound is the result of a more constrained model because the aggregated model only uses one unit in the blocks CFI, CFII, and CFIII instead of a combination of units in parallel.

In the aggregated model each block of centrifuges CFI, CFII, and CFIII is represented by only one centrifuge with the maximum inlet flowrate constraints relaxed. Note that the relaxations imposed in the maximum inlet flowrate of the centrifuges do not contribute to obtain a lower bound. However, this aggregation has two important implications: (1) in the detailed model in each block of centrifuges the units can operate at different temperatures, while in the aggregated model the aggregated centrifuge can only operate at a single temperature, (2) the performance of the aggregated unit can be different of the set of units combined in parallel. In the first point it is easy to understand that the lack of flexibility to operate the centrifuges at different temperatures may lead to a more constrained model and consequently to an upper bound. However, the second point needs to be explained in more detail. Here, the term performance is used to relate the output of a centrifuge with the same inlet flowrate.

Generally, the performance of a set of units in parallel is only equivalent to a single unit with an equivalent capacity if all the equations describing the units are linear. However, when the units are described by nonlinear equations, the performance of a set of units in parallel and one equivalent unit may not be the same. In Appendix A it is shown that the feasible region of the aggregated model is a subregion of the detailed model, and therefore the first yields an upper bound on the objective function.

\section{Integer cuts}

At each iteration of the two-level decomposition, one specific integer cut is added in each level in order to expedite the search. In the aggregated level an integer cut is implemented, with the goal of eliminating from the solution combinations of aggregated blocks with a previous equivalent number of centrifuges, where equivalent number of centrifuges denotes the number of centrifuges obtained from the ceiling of the ratio between the inlet flowrate and the maximum inlet flowrate of 
each centrifuge,

$$
\begin{aligned}
& N_{C F I}=\left\lceil\frac{F_{s S X}}{F_{s S X}^{U}}\right\rceil \quad \forall u \in U^{C F I}, \forall s \in S_{u}^{I} \\
& N_{C F I I}=\left\lceil\frac{F_{s}}{F_{s}^{U}}\right\rceil \quad \forall u \in U^{C F I I}, \forall s \in S_{u}^{I} \\
& N_{C F I I I}=\left\lceil\frac{F_{s S X}}{F_{s S X}^{U}}\right\rceil \quad \forall u \in U^{C F I I I}, \forall s \in S_{u}^{I}
\end{aligned}
$$

where $N_{C F I}, N_{C F I I}$ and $N_{C F I I I}$ are the equivalent number of required centrifuges to deal with the inlet streams, $F_{s S X}$ is the flowrate of solid p-xylene, $F_{s S X}^{U}$ is the upper bound on the inlet flowrate of solid p-xylene, $F_{s}$ is the total inlet flowrate and $F_{s}^{U}$ the upper bound of the total inlet flowrate.

Instead of using Equations 74 to 76, additional binary variables, $y_{u k}$, and disaggregated variables, $F_{s S X k}$ or $F_{s k}$, are introduced for each block to represent the actual number of units $k$. Figure 13 depicts the partition, in terms of these variables, for $F_{s S X}$, where the disaggregated variable $F_{s S X k}$ has as lower bound $F_{s S X}^{U}\left(k y_{u k}-1\right)+\varepsilon$, and upper bound $k y_{u k} F_{s S X}^{U}$.

\section{Figure 13}

This is represented mathematically by:

$$
\begin{aligned}
& F_{s S X}=\sum_{k=1}^{M} F_{s S X k} \quad \forall u \in U^{C F I}, \forall s \in S_{u}^{I} \\
& F_{s S X k} \leq F_{s S X}^{U} k y_{u k} \quad k=1, \ldots, M ; \forall u \in U^{C F I}, \forall s \in S_{u}^{I} \\
& F_{s S X k} \geq F_{s S X}^{U}\left(k y_{u k}-1\right)+\varepsilon \quad k=1, \ldots, M ; \forall u \in U^{C F I}, \forall s \in S_{u}^{I} \\
& \sum_{k=1}^{M} y_{u k}=1 \quad \forall u \in U^{C F I} \\
& F_{s}=\sum_{k=1}^{N} F_{s k} \quad \forall u \in U^{C F I I}, \forall s \in S_{u}^{I} \\
& F_{s k} \leq F_{s}^{U} k y_{u k} \quad k=0, \ldots, N ; \forall u \in U^{C F I I}, \forall s \in S_{u}^{I}
\end{aligned}
$$




$$
\begin{aligned}
& F_{s k} \geq F_{s}^{U}\left(k y_{u k}-1\right)+\varepsilon \quad k=1, \ldots, N ; \forall u \in U^{C F I I}, \forall s \in S_{u}^{I} \\
& \sum_{k=0}^{N} y_{u k}=1 \quad \forall u \in U^{C F I I} \\
& F_{s S X}=\sum_{k=1}^{P} F_{s S X k} \quad \forall u \in U^{C F I I I}, \forall s \in S_{u}^{I} \\
& F_{s S X k} \leq F_{s S X}^{U} k y_{u k} \quad k=1, \ldots, P ; \forall u \in U^{C F I I I}, \forall s \in S_{u}^{I} \\
& F_{s S X k} \geq F_{s S X}^{U}\left(k y_{u k}-1\right)+\varepsilon \quad k=1, \ldots, P ; \forall u \in U^{C F I I I}, \forall s \in S_{u}^{I} \\
& \sum_{k=1}^{P} y_{u k}=1 \quad \forall u \in U^{C F I I I} \quad \forall
\end{aligned}
$$

where $M, N$ and $P$ are the maximum number of units in each aggregated block CFI, CFII and CFIII, respectively, $F_{s S X k}$ and $F_{s k}$ are disaggregated variables, $y_{u k}$ are binary variables and $\varepsilon$ is a small number. Thus, for all blocks the integer cut is defined by the following expression:

$$
\sum_{u \mid \bar{y}_{u}^{K} \in P^{K}} y_{u}^{K}+\sum_{e \mid \bar{y}_{e}^{K} \in P^{K}} y_{e}^{K}-\sum_{u \mid \bar{y}_{u}^{K} \in N^{K}} y_{u}^{K}-\sum_{e \mid \bar{y}_{e}^{K} \in N^{K}} y_{e}^{K} \leqslant\left|P^{K}\right|-1
$$

where

$P^{K}=\left(\left\{u \mid \bar{y}_{u}^{K}=1\right\} \cup\left\{e \mid \bar{y}_{e}^{K}=1\right\}\right), N^{K}=\left(\left\{u \mid \bar{y}_{u}^{K}=0\right\} \cup\left\{e \mid \bar{y}_{e}^{K}=0\right\}\right)$ with $\left\{u \mid u \in U^{C R T} \cup U^{S L D}\right\}$ and $e=(l k)$ with

$$
\left\{l k:\left(l \in U^{C F I}, k=1, \ldots, j\right) \cup\left(l \in U^{C F I I}, k=1, \ldots, m\right) \cup\left(l \in U^{C F I I I}, k=1, \ldots, n\right)\right\}
$$

and $K$ denotes the iteration number of the two-level decomposition. Therefore, despite the fact that for each aggregated unit of centrifuges there is only one unit, an integer cut can be used to eliminate solutions with previous equivalent number of centrifuges.

The second cut was applied at the detailed level to avoid the repetition of previous combinations of units in the solution. In this case the integer cut is defined by Equation 90, associated with 
Equations 91, 92 and 93.

$$
\sum_{i||_{u}^{K} \in P^{K}} y_{u}^{K}-\sum_{i \mid \bar{y}_{u}^{K} \in N^{K}} y_{u}^{K} \leqslant\left|P^{K}\right|-1
$$

where $P^{K}=\left\{u \mid \bar{y}_{u}^{K}=1\right\}, N^{K}=\left\{u \mid \bar{y}_{u}^{K}=0\right\},\left\{u: u \in\left(U^{C F I} \cup U^{C F I I} \cup U^{C F I I I}\right)\right\}$ and $K$ denotes the iteration number of the two-level decomposition. In addition, to avoid symmetric solutions inside of each block of centrifuges the following equations were considered:

$$
\begin{array}{ll}
y_{k} \geq y_{k+1} ; & k=1, \ldots, M-1 \\
y_{k} \geq y_{k+1} ; & k=1, \ldots, N-1 \\
y_{k} \geq y_{k+1} ; & k=1, \ldots, P-1
\end{array}
$$

which set an order of selection for the units in parallel.

In the aggregated model the logic relationships between the binary variables assigned to each block, $y_{u}$ and the disaggregated binary variables is as follows:

$$
\begin{array}{cc}
\bigvee_{k=1, \ldots, M} y_{u k} \Leftrightarrow y_{u} & \forall u \in U^{C F I} \\
\bigvee_{k=1, \ldots, N} y_{u k} \Leftrightarrow y_{u} & \forall u \in U^{C F I I} \\
\bigvee_{k=1, \ldots, P} y_{u k} \Leftrightarrow y_{u} & \forall u \in U^{C F I I I}
\end{array}
$$

Transforming these logic propositions into inequalities yields ${ }^{24}$ :

$$
\begin{aligned}
& y_{u}-y_{u k} \geq 0 \quad \forall u \in U^{C F I}, k=1, \ldots, M \\
& \sum_{k=1}^{M} y_{u k}-y_{u} \geq 0 \quad \forall u \in U^{C F I}
\end{aligned}
$$




$$
\begin{aligned}
& y_{u}-y_{u k} \geq 0 \quad \forall u \in U^{C F I I}, k=1, \ldots, N \\
& \sum_{k=1}^{N} y_{u k}-y_{u} \geq 0 \quad \forall u \in U^{C F I I} \\
& y_{u}-y_{u k} \geq 0 \quad \forall u \in U^{C F I I}, k=1, \ldots, P \\
& \sum_{k=1}^{P} y_{u k}-y_{u} \geq 0 \quad \forall u \in U^{C F I I}
\end{aligned}
$$

\section{Initialization of the detailed model}

In the proposed decomposition approach, the aggregated model describes the superstructure defined in Figure 2. The optimization of the aggregated model provides solutions with several zero flowrates. This information is used to define a new superstructure for the detailed model, where the streams with zero flowrate in the aggregated model are removed from the superstructure of the detailed model. Therefore, an analysis of the input and output streams of splitters and mixers is made in order to remove from the detailed model the splitters with only one output stream and mixers with only one input stream. In this way several additional equations and variables are removed from the detailed model, decreasing considerably the size of the problem. In addition, singularities that may appear due to many zero entries in the Jacobian matrix are avoided. ${ }^{27}$ Figure 14 illustrates the reduction in terms of units and streams that can occur with this model reduction. In the detailed model the variables associated with the streams represented in Figure 2 are initialized using the values of the final solution of the aggregated model. However, it was necessary to devise an initialization strategy for the continuous and binary variables associated with the streams and units inside the blocks CFI, CFII, and CFIII.

\section{Figure 14}

As a first approach, based on the solution of the aggregated model, the number of units of blocks CFI, CFII, and CFIII (calculated by Equations 74 to 76) were set as the maximum number of units in the detailed model. However, due to nonlinearities this restriction has shown to eliminate some combinations of units from better solutions. Therefore, three alternative approaches 
were considered, where the main idea is to avoid starting points with sets of variables with zero values that could cause problems to the solvers. The first alternative approach consists of using the maximum number of units in each block, initialize each binary variable associated with each unit and divide the feed stream equally to each unit. The second alternative approach consists in using the values of units calculated by Equations 74 to 76, and divide $99 \%$ of the feed stream to those units, with only those initiated with $y_{u}=1$, while the remaining units share $1 \%$ of the feed stream and $y_{u}=0$. The third alternative divides the flowrate as in the second but all the binary variables were initialized with $y_{u}=1$.

\section{Decomposition strategy}

The steps of the suggested decomposition are as follows:

Step 1. Set $K=1$ and $K_{\max }$. Set $Z_{U}=\infty$ and $Z_{L}=-\infty$.

Step 2. Aggregate centrifuges in blocks, redefine new input and output streams of the aggregated blocks. Select an initial starting point and flowsheet through $y_{u k}$ and $y_{u}$.

Step 3. Solve the aggregated MINLP model to yield $Z_{U}^{K}$. If $Z_{U}^{K} \leq Z_{U}$ then $Z_{U}=Z_{U}^{K}$.

Step 4. Add the integer cut from Equation 89.

Step 5. Remove streams, splitters, mixers, drums, heat exchangers and crystallizers not used in the solution of the aggregated model. Define new input streams into units due to the elimination of streams and some units. Disaggregate the units in each centrifuge block using either of the three alternative approaches. Set the starting point with the solution from the aggregated model.

Step 6. Solve the detailed MINLP to yield $Z_{L}^{K}$. IF $Z_{L}^{K} \geq Z_{L}$ then $Z_{L}=Z_{L}^{K}$.

Step 7. Add the integer cut from Equation 90. 
Step 8. If $\left\{\exists u\left|y_{u}=1, F_{s}=0,\left\{u: u \in\left(U^{C F I} \cup U^{C F I I} \cup U^{C F I I I}\right)\right\}, s\right| s \in S_{u}^{I}\right\}$ add an integer cut (Equation 90), update $y_{u}$ and $Z_{L}^{K}$, and add one more integer cut (Equation 90) to the detailed model.

Step 9. If $Z_{L} \geq Z_{U}$ or $K>K_{\max }$ then $Z^{*}=Z_{L}$, STOP. Otherwise go to 3 .

\section{Remarks}

1. In the aggregated model the variables and the equations associated with the streams not present are not considered; i.e. they are eliminated from the model, instead of setting some binary variables to zero.

2. In the detailed model the only variables that cannot be initialized using the values from the aggregated model correspond to the variables associated with some streams of slurry drum blocks. All other variables can be initialized from the aggregated model solution.

3. Both MINLP models, aggregated and detailed, are nonconvex and have multiple suboptimal solutions. At each iteration of the two-level decomposition the MINLP problem is not solved to global optimality, which may lead to situations where the solution of the detailed model could be worse than the solution of the aggregated model.

4. Both integer cuts, Equations 89 and 90 are added to avoid the repetition of previous configurations. These integer cuts complement the cuts applied within DICOPT ${ }^{26}$, since the last are applied to all integer variables of the problem, while cuts imposed by the decomposition are only applied to the integer variables related with the process units. The integer cut added to the detailed model, is expected to increase the lower bound of the objective function, thus a stopping criterion was developed based on the crossing of upper and lower bound. In addition, a criterion based on the maximum number of iterations of the decomposition was implemented.

5. Deterministic algorithms for global optimization, such as BARON ${ }^{28}$ and LINDOGlobal ${ }^{29}$ were not able to be solved in many hours. 


\section{Numerical results}

The proposed superstructure and decomposition strategy were applied to three cases with different p-xylene compositions in the feed stream: Case I with 65 wt \%, Case II with 90 wt \% and Case III with $98 \mathrm{wt} \%$. For all cases the product specification is a stream with a flowrate of $13.8 \mathrm{~kg} / \mathrm{s}$ and 99.8 wt \% of p-xylene. These compositions were taken from Hubbell and Rutten ${ }^{3}$, who have proposed different flowsheets for each one. With these cases we pretend to cover the more recent trend where the crystallization is used as a second stage in the p-xylene recovery. Therefore, the inlet feed stream can be the output of an adsorption process. ${ }^{3}$

Note that the same prices for the feed and rejected filtrate are considered in the three aforementioned cases. This means that a feed with 65 wt $\%$ and another with 98 wt $\%$ p-xylene have the same economic value. Although this may not be accurate, the feed costs and rejected filtrate costs only influence the value of the objective function and do not have any influence in the structure of the optimum flowsheet. This was supported by several optimizations using different cost parameters.

The MINLP models and the decomposition strategy were implemented using GAMS ${ }^{25}$ and solved on a computer running Linux with a Intel Xeon CPU, 1.86GHz and 8GB of RAM. The strategy used to solve each MINLP problem involved two steps. First, GAMS/SBB was used to solve the MINLP problem with a maximum number of nodes, and then the output solution from GAMS/SBB was used as an initial point for GAMS/DICOPT. The aim of this strategy was to perform a fast screening in the branch and bound tree, and then to try to improve the solution with GAMS/DICOPT. In this way most of the times GAMS/DICOPT started with a feasible integer solution.

Table 2 shows the sizes of the aggregated model and typical sizes of the detailed models for Case III. Note that while the size of the aggregated model is fixed for the three cases, the size of the detailed model can vary depending on the solution of the aggregated model.

Table 2 
As can be seen, the proposed decomposition scheme requires a significantly smaller sized MINLP.

\section{Case I}

The optimum flowsheet obtained for the first case, with a process feed stream with $65 \mathrm{wt} \%$ of p-xylene, had a cost of 101.2 m.u./year and is shown in Figure 15.

\section{Figure 15}

In the optimum flowsheet the blocks CSII and SLDII are not included, and only two crystallizers are used in the block CSI. The process feed stream is divided into two streams that are mixed with several recycle streams and feed to each crystallizer. Analyzing the input flowrate of the block of centrifuges CFII, the number of centrifuges do not correspond to the minimum number of centrifuges. This fact suggested that this solution may correspond to a local minimum. Therefore, this case was solved using the third alternative for the initialization. The best solution obtained corresponded to a flowsheet with three units in the block CFII, but with a worse value of the objective function, 101.4 m.u./year, (see Table 3). This shows the impact of the initialization of the streams of block CFII in the detailed model, but also that the minimum number of units in each block may not correspond to the minimum value of the objective function.

The p-xylene recovery rate was $90.3 \mathrm{wt} \%$, which is in agreement with the value of $90.7 \mathrm{wt} \%$ reported by Hubbell and Rutten ${ }^{3}$. Table 4 shows that the algorithm stopped after nine iterations because the bounds crossed with each other. The optimal solution was found in iteration number four, 101.2 m.u./year, where the operating costs corresponds to $94.6 \%$ of the total cost, with the feed stream contributing to $92.6 \%$ of the total cost, (see Table 7).

Table 3

Table 4

\section{Case II and III}

For the two cases with 90 wt \% and 98 wt \% of p-xylene in the process feed stream, the optimal flowsheets are shown in Figures 16a) and 16b), with costs of 99.5 m.u./year and 98.4 m.u./year, 
respectively.

\section{Figure 16}

These two flowsheets present considerable differences in terms of topology when compared with the flowsheet presented for Case I. The main features of these flowsheets are: (1) use of two crystallization stages at different temperature levels, where at the higher temperature level the process feed stream is cooled down until the upper bound on the temperature of the outlet stream, 234.4 $\mathrm{K}$, while at the lower temperature level the $\mathrm{p}$-xylene from the filtrates from the centrifuges is crystallized at the eutectic temperature, $205.7 \mathrm{~K}$; (2) the output stream from the warmer level is only directed to the block CFIII where it is mixed with different streams to meet the centrifuges temperature constraints; and (3) one centrifuge in the block CFI in both cases, and only one centrifuge in the block CFII in Case III.

The flowsheet presented for the $90 \mathrm{wt} \%$ case was obtained using the first alternative approach for the initialization. In this flowsheet the number of centrifuges used in the block CFII is also greater than the required number of centrifuges based on the total flowrate that they are treating. Therefore, in order to assess if a better solution with a lower number of centrifuges in this block was possible, the third initialization alternative was also employed. The optimum flowsheet obtained includes three units in the block CFII, but two more crystallizers leading to a similar objective function value, 100.3 m.u./year as shown in Table 3.

For these two cases the total annualized operating costs correspond to more than $97.2 \%$ of the total cost, where the cost of the feed contributing to $97.1 \%$ and $98.0 \%$ of the total cost, in Cases II and III, respectively, (see Table 7). For Case II the optimum solution was found at the second iteration and the decomposition stopped because the bounds cross with each other as seen in Table 5.

Table 5

Analyzing these results, it can be observed that in iteration three the lower bound, $Z_{L}^{K}$, is greater than the upper bound, $Z_{U}^{K}$. This may occur, as previously explained, because the detailed model got trapped in a local solution. 
Table 6 shows the results for each iteration of Case III, where it can observed that the decomposition stopped after two iterations because the bounds crossed with each other. In this case, in the second iteration the value of the lower bound, 98.4 m.u./year, is similar to the value of the upper bound. This can occur because in the solution of both models the blocks CFI and CFII only have one unit, and therefore the effect of units in parallel is not present here as discussed in Appendix A. Note also, that the algorithm did not stop in the first iteration, even with $Z_{L}^{K}>Z_{U}^{K}$, because the stop criteria is relaxed for the first iteration.

Table 6

The p-xylene recovery rates obtained for these processes were $97.4 \mathrm{wt} \%$ and $99.5 \mathrm{wt} \%$ for the feed streams with $90 \mathrm{wt} \%$ and $98 \mathrm{wt} \%$, respectively. The optimum flowsheets obtained in this work are similar to the ones proposed by Hubbell and Rutten ${ }^{3}$ and Wilsak ${ }^{11}$. For the feed streams with high concentrations of p-xylene they also suggest flowsheets where the feed stream is cooled down at a warmer level in a set of crystallizers and the output slurry is centrifuged, separating the filtrate from the desired high concentration p-xylene product. Their flowsheets include also another set of crystallizers to recover the p-xylene from the previously mentioned filtrate, and additional filtrates from centrifuges used to separate the output slurry from a second stage of crystallizers. However, due to the lack of information in these patents in terms of operating conditions and number of units, it is not possible to perform a detailed comparison.

In our superstructure all units have bounds on the inlet and outlet temperatures. These bounds not only constrain the feasible links between the units, but also the mixing of streams to meet constraints in the inlet streams of centrifuges. Therefore, different specifications for the inlet or outlet temperature streams can change the operating conditions and give rise to different flowsheets with alternative topologies.

Table 7 


\section{Conclusions}

In this paper we have proposed a mathematical programming approach for the optimal synthesis of p-xylene recovery based on crystallization. Our approach consists of the following three major steps: a) development of a superstructure for the crystallization and associated liquid/solid separation stages; b) formulation of an MINLP model associated with the superstructure; and c) solution of the MINLP model employing a decomposition approach.

The proposed superstructure includes several alternative flowsheets in order to be able to deal with compositions of p-xylene in the process feed stream ranging from $65 \mathrm{wt} \%$ to $98 \mathrm{wt} \%$.

The complexity of the superstructure and MINLP model motivated the study of a two-level decomposition approach that could cope with difficulties inherent with a nonconvex and large size model. The initialization and optimization of the aggregated model have proved to make it easier to solve the MINLP than the full size problem, while at the same time providing good starting points for the detailed model. Although the proposed decomposition approach does not guarantee global optimality, it provides a methodology to provide different starting points that can lead to different optimal solutions with different combinations of units.

For the three cases presented, the superstructure demonstrated enough flexibility to deal with pxylene compositions in the feed stream above $65 \mathrm{wt} \%$. The crystallization network, CSI, revealed to be able to cope with the existence of two crystallization stages at different temperatures. This flexibility could be used in the future to create an extended superstructure where an alternative separation process based on adsorption could be included in the superstructure.

The flowsheets and the results in terms of p-xylene recovery are in agreement with published results in the literature, which verifies the accuracy of the model.

Acknowledgments - The first author would like to acknowledge financial support from FCT (Portuguese Foundation for Science and Technology) under contract SFRH/BPD/26115/2005. 


\section{Nomenclature}

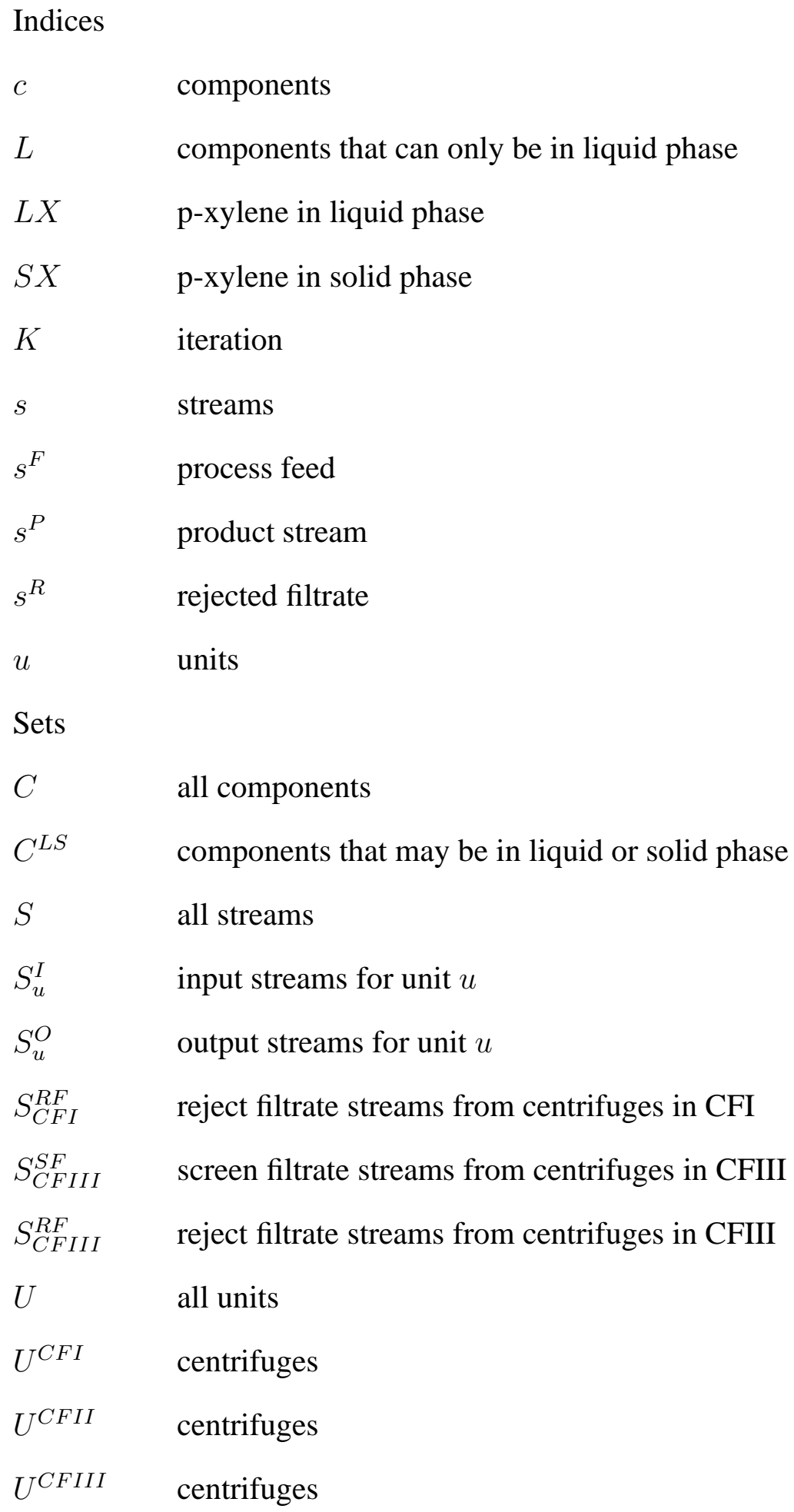




\begin{tabular}{|c|c|}
\hline$U^{C R T}$ & all crystallizers \\
\hline$U^{C S I}$ & only crystallizers in the first crystallization stage \\
\hline$U^{C S I I}$ & only crystallizers in the second crystallization stage \\
\hline$U^{H E H}$ & heat exchangers \\
\hline$U^{M X R}$ & mixers \\
\hline$U^{S L D}$ & slurry drums \\
\hline$U^{S P L}$ & splitters \\
\hline \multicolumn{2}{|c|}{ Parameters } \\
\hline$C_{E}$ & electricity cost, $\$ /(\mathrm{kWh})$ \\
\hline$C_{F D}$ & feed cost, $\$ \mathrm{~kg}^{-1}$ \\
\hline$C_{F L}$ & fuel cost, $\$ \mathbf{J}^{-1}$ \\
\hline$C_{H E}$ & hot end cost, \$ \\
\hline$C_{R}$ & rejected filtrate cost, $\$ \mathrm{~kg}^{-1}$ \\
\hline$C_{S}$ & steam cost, $\$ \mathrm{~kg}^{-1}$ \\
\hline$F_{s}^{L}$ & lower bound on flow in stream $s, \mathrm{~kg} \mathrm{~s}^{-1}$ \\
\hline$F_{s S X}^{L}$ & lower bound on the individual flow of component $S X$ in stream $s, \mathrm{~kg} \mathrm{~s}^{-1}$ \\
\hline$F^{\min }$ & minimum flowrate of the product stream, $\mathrm{kg} \mathrm{s}^{-1}$ \\
\hline$F_{s}^{U}$ & upper bound on flow in stream $s, \mathrm{~kg} \mathrm{~s}^{-1}$ \\
\hline$F_{s S X}^{U}$ & upper bound on the individual flow of component $S X$ in stream $s, \mathrm{~kg} \mathrm{~s}^{-1}$ \\
\hline$H C U_{u}$ & heat transfer coefficient of unit $\forall u \in U^{H E H}, \mathrm{~J} \mathrm{~s}^{-1} \mathrm{~m}^{-2} \mathrm{~K}^{-1}$ \\
\hline$I$ & maximum number of crystallizers in the block CSI \\
\hline$I_{2}$ & maximum number of crystallizers in the block CSII \\
\hline$M$ & number of units in block CFI \\
\hline$K$ & iteration number of the two-level decomposition \\
\hline
\end{tabular}




\begin{tabular}{|c|c|}
\hline$k$ & intervals \\
\hline$N$ & number of units in block CFII \\
\hline$P$ & number of units in block CFIII \\
\hline$T^{e u t}$ & lower bound on stream temperature, eutectic point, $\mathrm{K}$ \\
\hline$T^{i c t}$ & upper bound on the temperature of output streams of crystallizers, $\mathrm{K}$ \\
\hline$\eta$ & minimum concentration of $\mathrm{p}$-xylene in product feed, wt \% \\
\hline$\alpha_{u}$ & cost parameter \\
\hline$\beta_{u}$ & cost parameter \\
\hline$\epsilon$ & small value \\
\hline$\eta$ & minimum concentration of $\mathrm{p}$-xylene in product feed, wt \% \\
\hline$\zeta_{c}$ & concentration of each component $c$ in the feed stream, wt \% \\
\hline$\kappa_{u}$ & liquid/solid device parameter of unit $u$ \\
\hline$\nu_{u}$ & cost parameter \\
\hline$\xi_{u s}^{L}$ & lower bound on split fraction for stream $s$ in splitter $u$ \\
\hline$\xi_{u s}^{U}$ & upper bound on split fraction for stream $s$ in splitter $u$ \\
\hline \multicolumn{2}{|c|}{ Variables } \\
\hline$D_{u}$ & size of unit $u, \mathrm{~m}^{2}$ or $\mathrm{m}^{3}$ \\
\hline$E L E_{u}$ & electricity consumed by unit $u, \mathrm{kWh}$ \\
\hline$F_{s}$ & flowrate of the stream $s, \mathrm{~kg} \mathrm{~s}^{-1}$ \\
\hline$F_{s c}$ & flowrate of the component $c$ in stream $s, \mathrm{~kg} \mathrm{~s}^{-1}$ \\
\hline$F_{s L X}$ & flowrate of the liquid p-xylene in stream $s, \mathrm{~kg} \mathrm{~s}^{-1}$ \\
\hline$F_{s L}$ & flowrate of the aggregated component in stream $s, \mathrm{~kg} \mathrm{~s}^{-1}$ \\
\hline$F_{s S X}$ & flowrate of the solid p-xylene in stream $s, \mathrm{~kg} \mathrm{~s}^{-1}$ \\
\hline$F_{s^{P} c}$ & flowrate of the component $c$ in the product feed, $\mathrm{kg} \mathrm{s}^{-1}$ \\
\hline
\end{tabular}


$F_{s^{P} L X} \quad$ flowrate of the liquid $\mathrm{p}$-xylene in the product feed, $\mathrm{kg} \mathrm{s}^{-1}$

$F_{s^{P} S X} \quad$ flowrate of the solid p-xylene in the product feed, $\mathrm{kg} \mathrm{s}^{-1}$

$F_{s^{R}} \quad$ flowrate of the rejected filtrate, $\mathrm{kg} \mathrm{s}^{-1}$

$F_{s^{R} L X} \quad$ flowrate of the liquid p-xylene in the rejected filtrate, $\mathrm{kg} \mathrm{s}^{-1}$

$F_{S^{R} S X} \quad$ flowrate of the solid $\mathrm{p}$-xylene in the rejected filtrate, $\mathrm{kg} \mathrm{s}^{-1}$

$F D \quad$ flowrate of the feed to the process, $\mathrm{kg} \mathrm{s}^{-1}$

$F D_{c} \quad$ flowrate of the component $c$ in the process feed, $\mathrm{kg} \mathrm{s}^{-1}$

$F L_{s} \quad$ liquid flowrate of stream $s, \mathrm{~kg} \mathrm{~s}^{-1}$

$H A_{s} \quad$ heat added in heat exchangers to stream $s, \mathrm{~J} \mathrm{~s}^{-1}$

$H R_{s} \quad$ heat removed in crystallizers from stream $s, \mathrm{~J} \mathrm{~s}^{-1}$

$H T C_{u} \quad$ heat transfer coefficient of unit $\forall u \in U^{C R T}, \mathrm{~J} \mathrm{~s}^{-1}$

$P X_{O F F S E T}$ concentration of p-xylene in the rejected filtrate stream, wt $\%$

$T_{s} \quad$ temperature of stream $s, \mathbf{K}$

$T_{u} \quad$ operating temperature of unit $u, \mathrm{~K}$

$Z \quad$ total annualized cost, $\$$ year $^{-1}$

$\Delta T_{1} \quad$ temperature difference 1 for LMTD, $\mathrm{K}$

$\Delta T_{2} \quad$ temperature difference 2 for LMTD, $\mathrm{K}$

$\varepsilon_{s} \quad$ amount of component required to meet solubility, $\mathrm{kg} \mathrm{s}^{-1}$

$\varepsilon_{s}^{1} \quad$ disaggregated variable for $\varepsilon_{s}, \mathrm{~kg} \mathrm{~s}^{-1}$

$\varepsilon_{s}^{2} \quad$ disaggregated variable for $\varepsilon_{s}, \mathrm{~kg} \mathrm{~s}^{-1}$

$\xi_{u s} \quad$ split fraction of stream $s$ in splitter $u$

$\rho_{s} \quad$ density of the stream $s, \mathrm{~kg} \mathrm{~m}^{-3}$

$\sigma_{s} \quad$ solubility prediction for stream $s$, wt $\%$

Binary variables 
$y_{\varepsilon 1} \quad$ equal to 1 if variable $\varepsilon_{s}^{1}$ takes a value between 0 and $\varepsilon_{s}^{U}$

$y_{\varepsilon 2} \quad$ equal to 1 if variable $\varepsilon_{s}^{2}$ takes a value between $\varepsilon_{s}^{L}$ and 0

$y_{u} \quad$ equal to 1 if unit $u$ is present

$y_{u k} \quad$ equal to 1 if $F_{s c^{s x} k}$ is between $F_{s S X}^{U}\left(k y_{u k}-(1-\varepsilon)\right)$ and $k F_{s S X}^{U} y_{u k}$

$z \quad$ equal to 1 if exits at least one crystallizer in the second crystallization stage

$z_{1} \quad$ equal to 1 if stream 913 exists and stream 914 does not

$z_{2} \quad$ equal to 1 if stream 915 exists and stream 916 does not 


\section{References}

1. Cannella W. Kirk-Othmer Encyclopedia of Chemical Technology, chapter Xylenes and ethylbenzene. John Wiley \& Sons. 2001;

2. Minceva M, Rodrigues AE. Understanding and revamping of industrial scale SMB units for p-xylene separation. AIChE J. 2007;53:138 - 149.

3. Hubbell D, Rutten P. Crystallization process for purification of paraxylene. United States Patent No. 5,811,629. 1998.

4. Spannagel H, Tschunkar E. Separation and purification of ortho, meta and para xylene. United States Patent No. 1,940,065. 1933.

5. Spiller Jr Ca. Paraxylene purification system. United States Patent No. 2,866,833. 1958.

6. Lammers G. Process for the recovery of paraxylene. United States Patent No. 3,177,265. 1965.

7. Laurich S. p-xylene process. United States Patent No. 3,467,724. 1969.

8. Lindley J, McLeod A. Separation process by fractional crystallization. United States Patent No. 3,959,978. 1976.

9. Eccli W, Fremuth A. Single temperature stage crystallization of paraxylene. United States Patent No. 5,498,822. 1996.

10. Mikitenko P, MacPherson S. Process for separating paraxylene comprising at least two crystallization stages at high temperature. United States Patent No. 6,147,272. 2000.

11. Wilsak R. Energy efficient process for producing high purity paraxylene. United States Patent No. 6,565,653. 2003.

12. Daichendt M, Grossmann I. Integration of hierarchical decomposition and mathematical programming for the synthesis of process flowsheets. Comput Chem Eng. 1998;22:147-175.

13. Lin SW, Ng KM, Wibowo C. Synthesis of crystallization processes for systems involving solid solutions. Comput Chem Eng. 2008;32:956-970.

14. Lin $\mathrm{S}, \mathrm{Ng} \mathrm{K}$, Wibowo $\mathrm{C}$. A visual approach for integrating chemistry research and process design: Separation process with crystallization steps. Ind Eng Chem Res. 2005;44:6233- 
6245.

15. Wibowo C, Ng K. Unified approach for synthesizing crystallization-based separation processes. AIChE J. 2000;46:1400-1421.

16. Wibowo C, Chang W, Ng K. Design of integrated crystallization systems. AIChE J. 2001; 47:2474-2492.

17. Chang W, Ng K. Synthesis of processing system around a crystallizer. AIChE J. 1998; $44: 2240-2251$.

18. Cisternas LA, Vasquez CM, Swaney RE. On the design of crystallization-based separation processes: Review and extension. AIChE J. 2006;52:1754 - 1769.

19. Grossmann IE, Caballero JA, Yeomans H. Advances in mathematical programming for the synthesis of process systems. Latin Am Appl Res. 2000;30:263 - 284.

20. Cisternas L, Cueto J, Swaney R. Flowsheet synthesis of fractional crystallization processes with cake washing. Comput Chem Eng. 2004;28:613 - 623.

21. Méndez C, Myers J, Roberts S, Logdson J, Vaia A, Grossmann I. MINLP model for synthesis of paraxylene separation processes based on crystallization technology. In: L Puigjaner (Ed.) European Symposium on Computer Aided Process Engineering - 15, Elsevier.

22. Balakrishna S, Biegler LT. Targeting strategies for the synthesis and energy integration of nonisothermal reactor networks. Ind Eng Chem Res. 1992;31:2152 - 2164.

23. Balas E. Disjunctive programming and a hierarchy of relaxations for discrete optimization problems. SIAM J Algebra Discr. 1985;6:466-486.

24. Raman R, Grossmann IE. Relation between milp modeling and logical inference for chemical process synthesis. Computers \& Chemical Engineering. 1991;15:73 - 84 .

25. Brooke A, Kendrick D, Meeraus A, Raman R. GAMS - A user's guide. 1998, URL http: //www.gams.com.

26. Viswanathan J, Grossmann IE. A combined penalty-function and outer-approximation method for MINLP optimization. Comput Chem Eng. 1990;14:769 - 782.

27. Kocis GR, Grossmann IE. A modeling and decomposition strategy for the MINLP optimiza- 
tion of process flowsheets. Comput Chem Eng. 1989;13:797 - 819.

28. Tawarmalani M, Sahinidis N. Global optimization of mixed-integer nonlinear programs: A theoretical and computational study. Mathematical Programming. 2004;99:563-591.

29. Lindo systems I. LINDOGlobal. 2007. 


\section{Appendix A}

Consider two separation systems to perform the same task involving the same type of units. These units have one input and two output streams. The first system is composed by only one unit, and the second system involves three units in parallel, (see Figure 17).

\section{Figure 17}

The first system is described by the set of equations, $h\left(x_{0}, z, u\right)=0$,

$$
\begin{aligned}
& x_{0}=z+u \\
& z=f\left(x_{0}\right)
\end{aligned}
$$

and the second by, $g\left(x_{0}, z, u_{i}, x_{i}, y_{i}\right)=0$,

$$
\begin{aligned}
& x_{0}=x_{1}+x_{2}+x_{3} \\
& z^{\prime}=z_{1}+z_{2}+z_{3} \\
& u^{\prime}=u_{1}+u_{2}+u_{3} \\
& z_{i}=f\left(x_{i}\right), \quad i=1,2,3
\end{aligned}
$$

where $f(x)$ is the relation between the input and one output stream, with $f(0)=0$. Let $\mathrm{F}_{A}$ be the feasible region of the first system,

$$
\mathrm{F}_{A}=\left\{\left(x_{0}, z, u\right) \mid h=0, x_{0}, z, u \geq 0\right\}
$$

and $\mathrm{F}_{D}$ the feasible region of the second system

$$
\mathrm{F}_{D}=\left\{\left(x_{0}, z^{\prime}, u^{\prime}, x_{i}, y_{i}, u_{i}\right) \mid g=0, x_{0}, z^{\prime}, u^{\prime}, x_{i}, y_{i}, u_{i} \geq 0\right\}
$$


Proposition: $\mathrm{F}_{A} \subseteq \mathrm{F}_{D}$ for nonlinear $f(x)$.

Proof: Assume that $\mathrm{F}_{D} \subset \mathrm{F}_{A}$. This implies $\exists z \in \mathrm{F}_{A}, z \notin \mathrm{F}_{D}$. First, from Equations A.4 and A.6 we have $z^{\prime}=\sum_{i=1}^{3} f\left(x_{i}\right)$, and for $x_{i} \neq 0$ and $x_{j}=0, \forall j \neq i$ it follows that $f\left(x_{i}\right)=f\left(x_{0}\right)$ and $z^{\prime}=z$. However, for $x_{i} \neq 0, \forall i, z^{\prime}$ is equal or different to $z$. For linear $f(x), z^{\prime}=z$, but for nonlinear $f(x)$, it is generally different. Therefore, $\exists z^{\prime} \in \mathrm{F}_{D}$ such that $z^{\prime} \notin \mathrm{F}_{A}$, which contradicts the assumption $\mathrm{F}_{D} \subset \mathrm{F}_{A}$. Hence, $\mathrm{F}_{A} \subseteq \mathrm{F}_{D}$.

Corollary: Applying the above proposition for the blocks of centrifuges in the proposed models, the feasible region of the aggregated model represents a more constrained region than the region of the detailed model. Therefore, the aggregated model yields an upper bound on the objective function. 


\section{List of Figures}

Figure 1 Simplified crystallization separation process with main units.

Figure 2 Proposed superstructure, delimited by the discontinuous line. PEZ- p-xylene enriching zone. CSI and CSII - crystallization networks. CFI, CFII, and CFIII- centrifuge networks. SLDI and SLDII- slurry drum networks. HEH6- heat exchanger.

Figure 3 Proposed crystallization networks corresponding to blocks CSI and CSII in Figure 2. $I$ and $I_{2}$ denote the maximum number of crystallizers in CSI and CSII, respectively.

Figure 4 Embedded networks of centrifuges in blocks CFI, CFII, and CFIII in Figure 2. $M$, $N$ and $P$ denote the maximum number of units in blocks CFI, CFII, and CFIII, respectively.

Figure 5 Embedded networks of slurry drums in blocks SLDI and SLDII in Figure 2.

Figure 6 Mole fraction of p-xylene in the solution as a function of the temperature. (-) Using van Hoff relationship, (- -) employing cryoscopic constants.?

Figure 7 Second crystallization stage extracted from the proposed superstructure.

Figure 8 Units around the heat exchanger located before the second crystallization stage, extracted from the proposed superstructure.

Figure 9 Set of centrifuges CFI from the detailed model (left) and the respective structure in the aggregated model (right).

Figure 10 Superstructure for a block of centrifuges from the detailed model (left) and the respective structure in the aggregated model (right).

Figure 11 Superstructure for a block of centrifuges from the detailed model (left) and the respective structure in the aggregated model (right).

Figure 12 Two-level decomposition approach consisting of the solution of an aggregated and detailed model.

Figure 13 Partition for the variable $F_{s S X}$ based on the variables $F_{s S X k}$ and $y_{u k}$.

Figure 14 Example of the analysis that is made in order to remove streams and mixers from the detailed model based on the results from the aggregated model.

Figure 15 Optimum flowsheets obtained for a feed stream with 65 wt \% p-xylene. CFI, CFII, and CFIII - centrifuges, CRT - crystallizers, SLD - reslurry drums.

Figure 16 Optima flowsheets obtained for different compositions of p-xylene in the feed stream. 
CFI, CFII, and CFIII - centrifuges, CRT - crystallizers, SLD - reslurry drums.

Figure 17 a) System with one unit, and b) system with three units in parallel. 


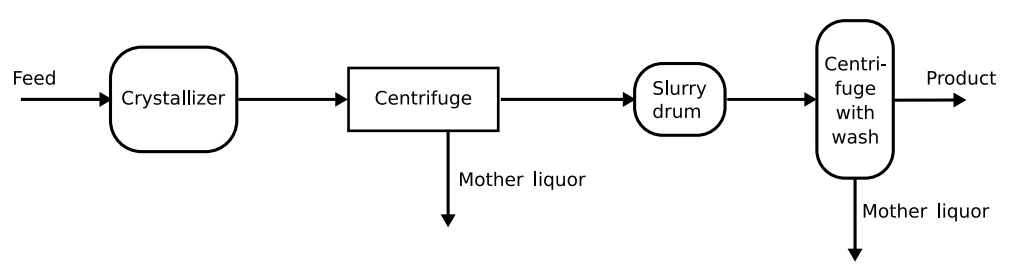

Figure 1 


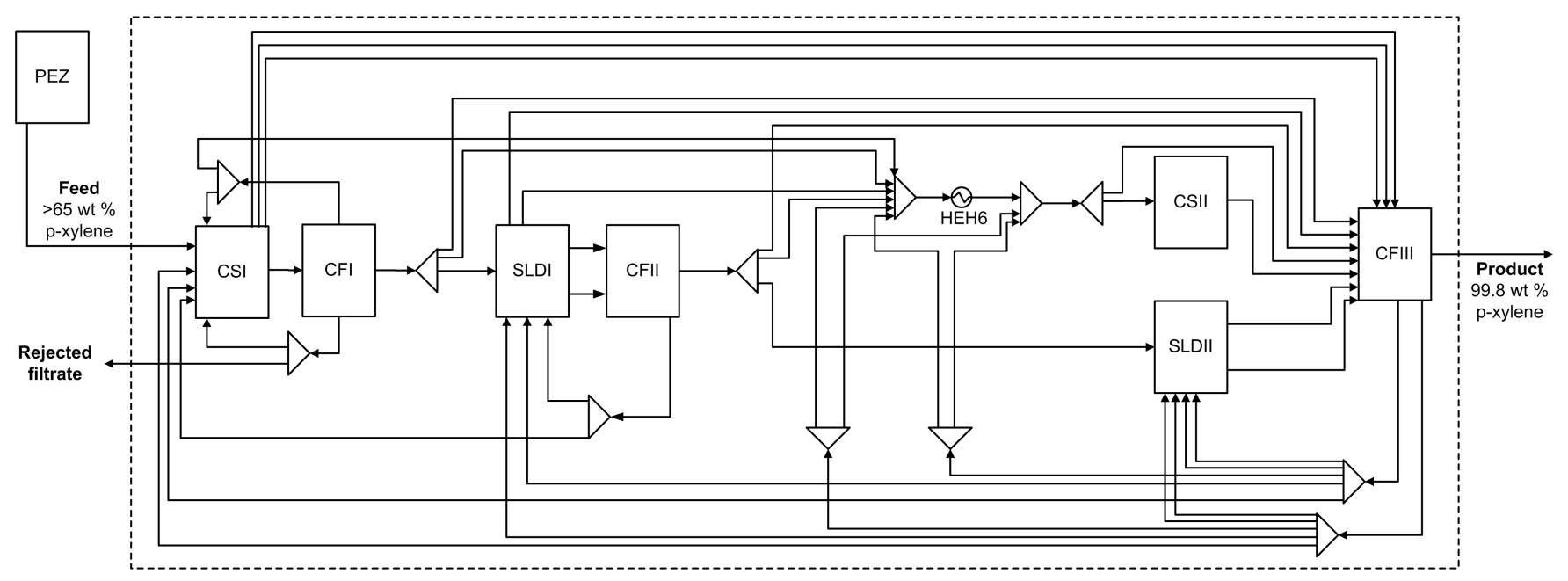

Figure 2 


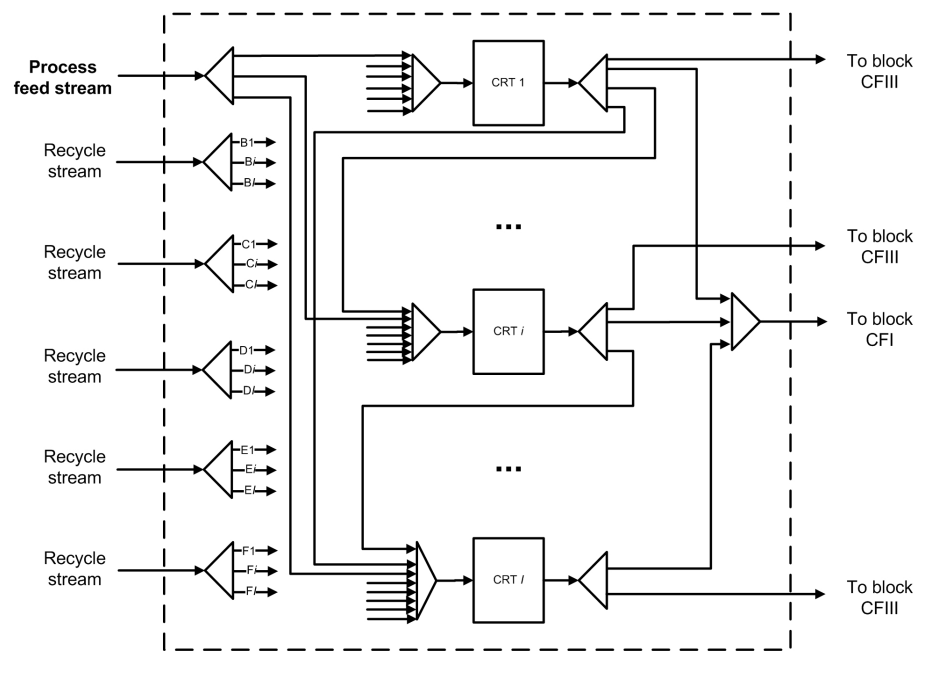

a) Block CSI.

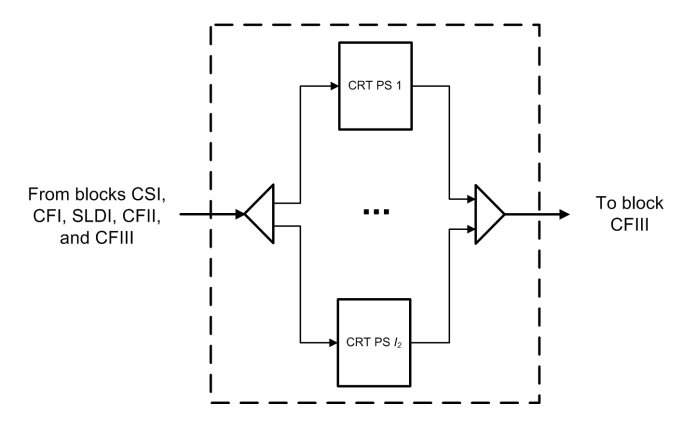

b) Block CSII.

Figure 3 


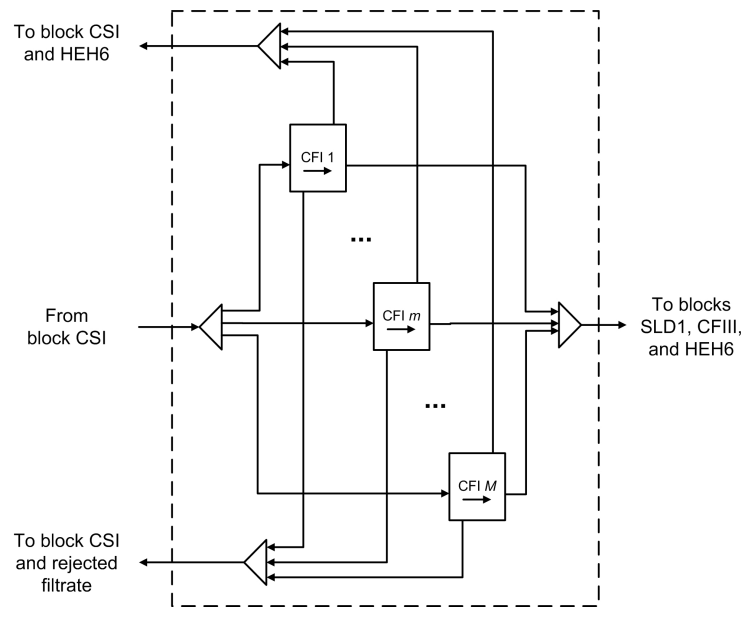

a) Structure within block CFI in Figure 2 .

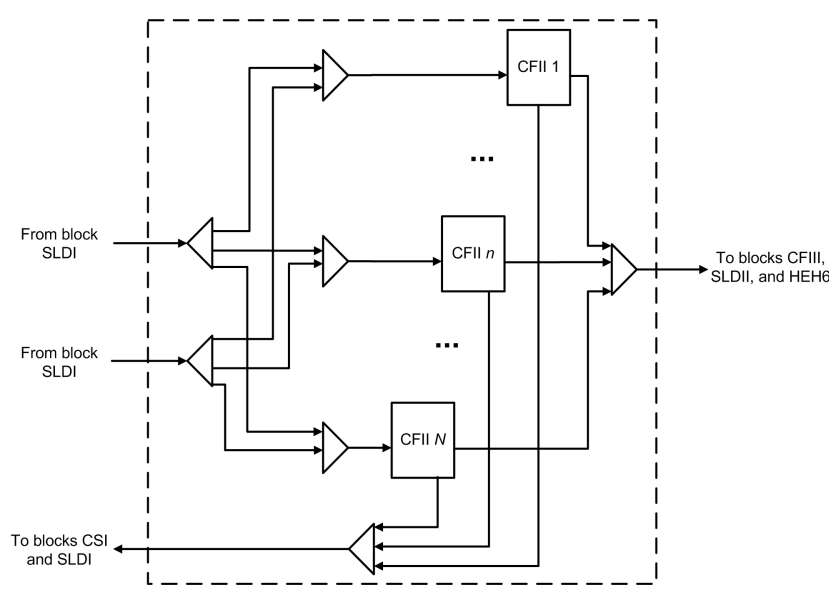

b) Structure within block CFII in Figure 2.

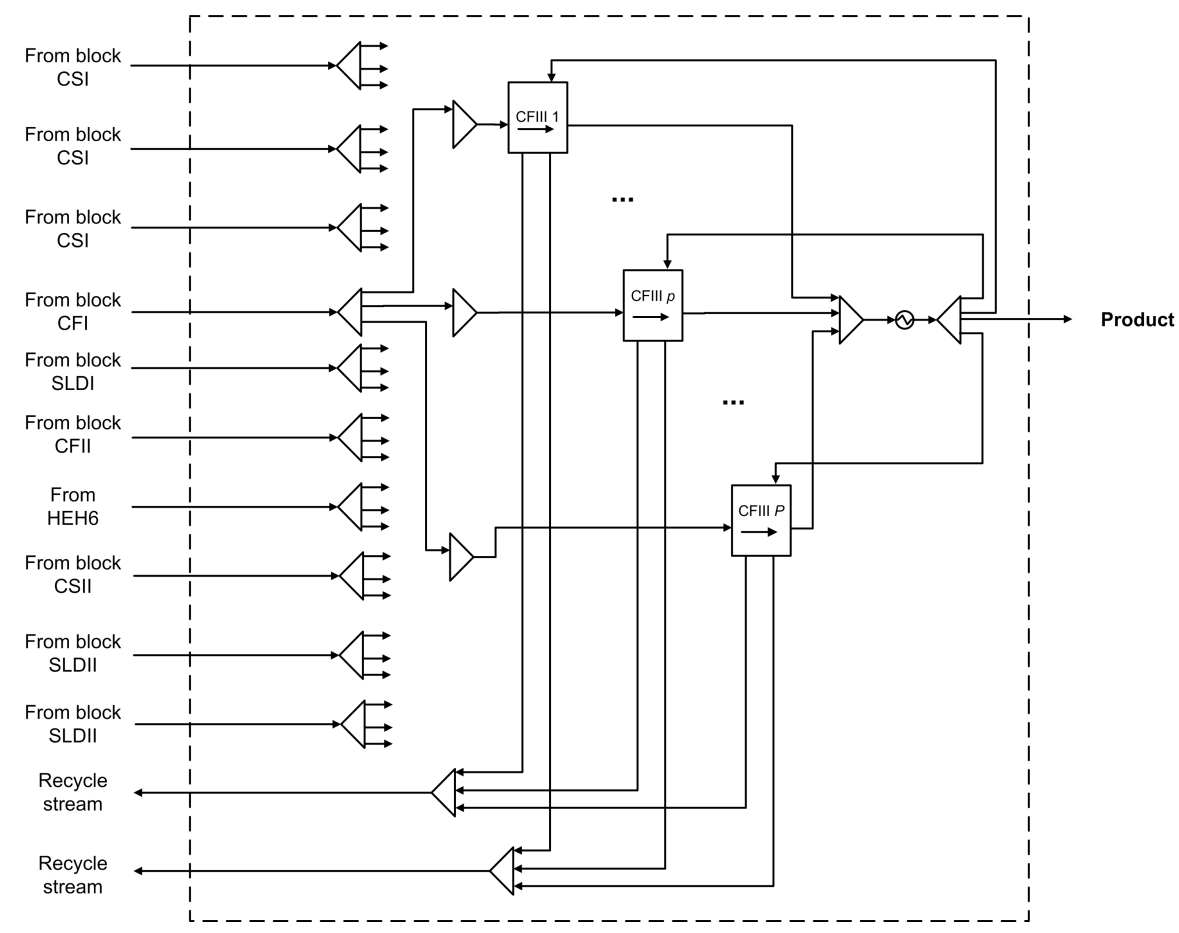

c) Structure within block CFIII in Figure 2. Note that for the sake of simplicity the streams from the splitters on the left are not connected to the mixers before the centrifuges.

Figure 4 


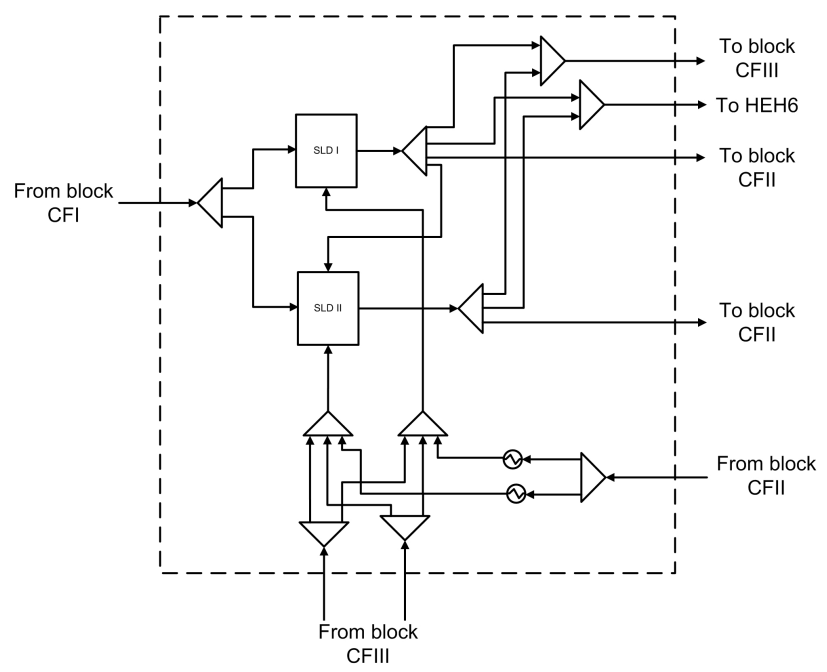

a) Structure within block SLDI.

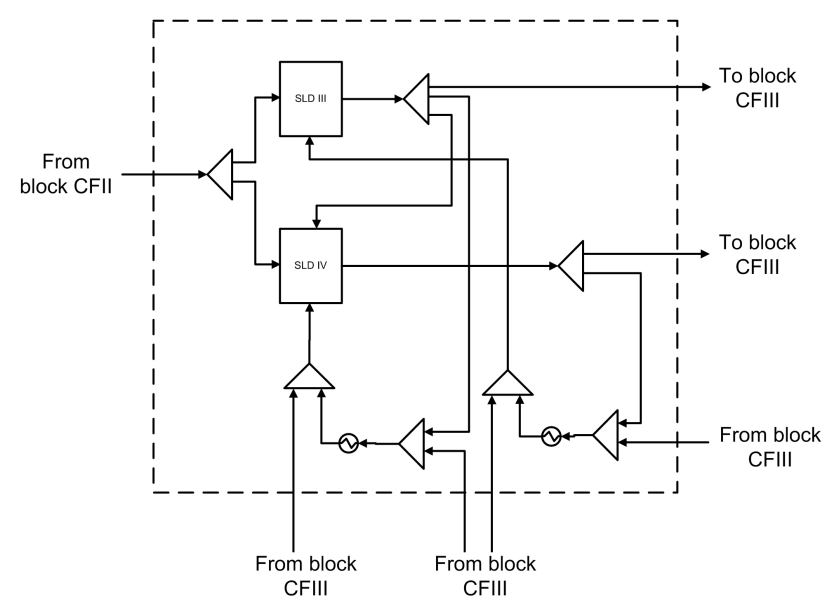

b) Structure within block SLDII.

Figure 5 


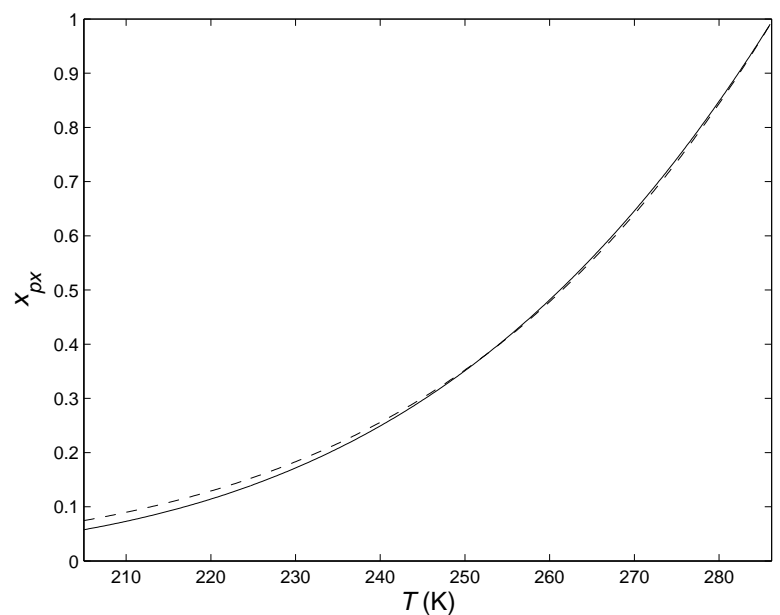

Figure 6 


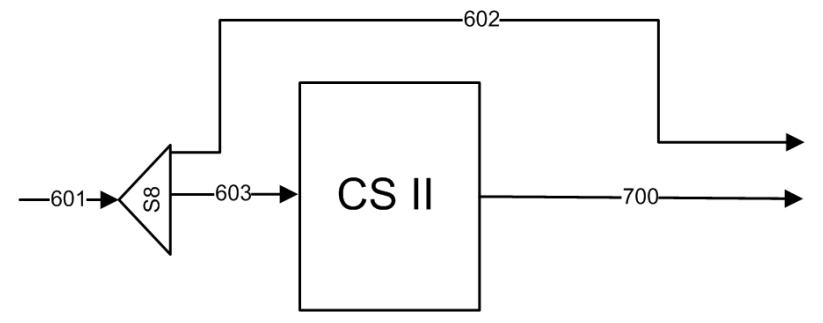

Figure 7 


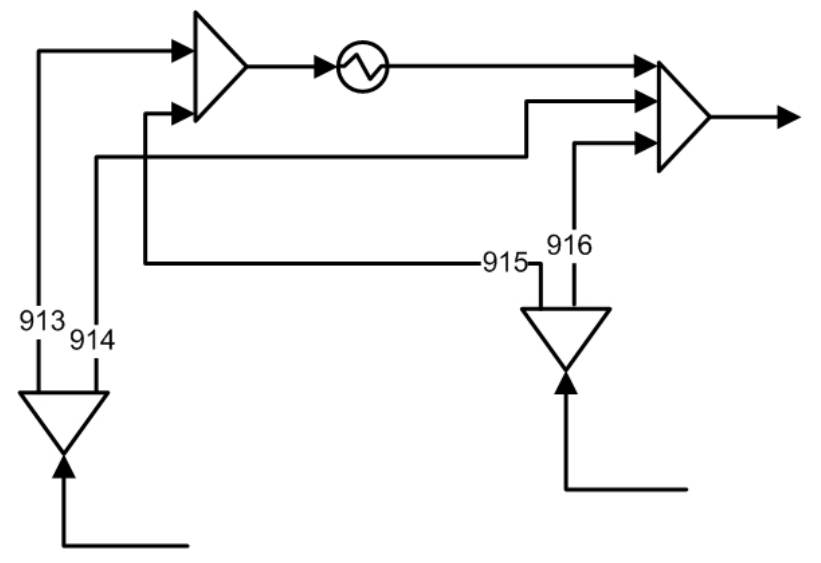

Figure 8 


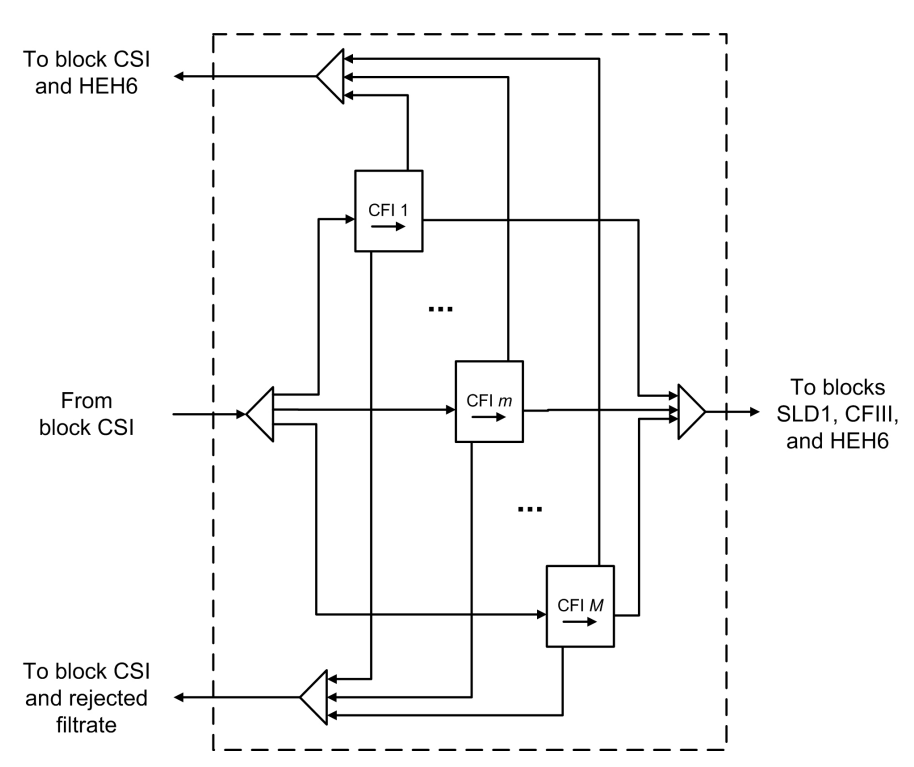

a) Detailed model.

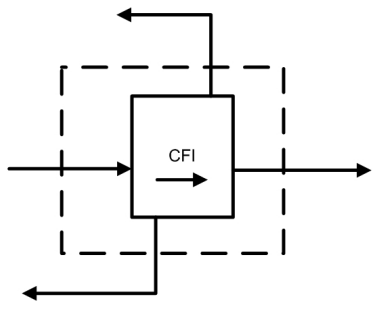

b) Aggregated model.

Figure 9 


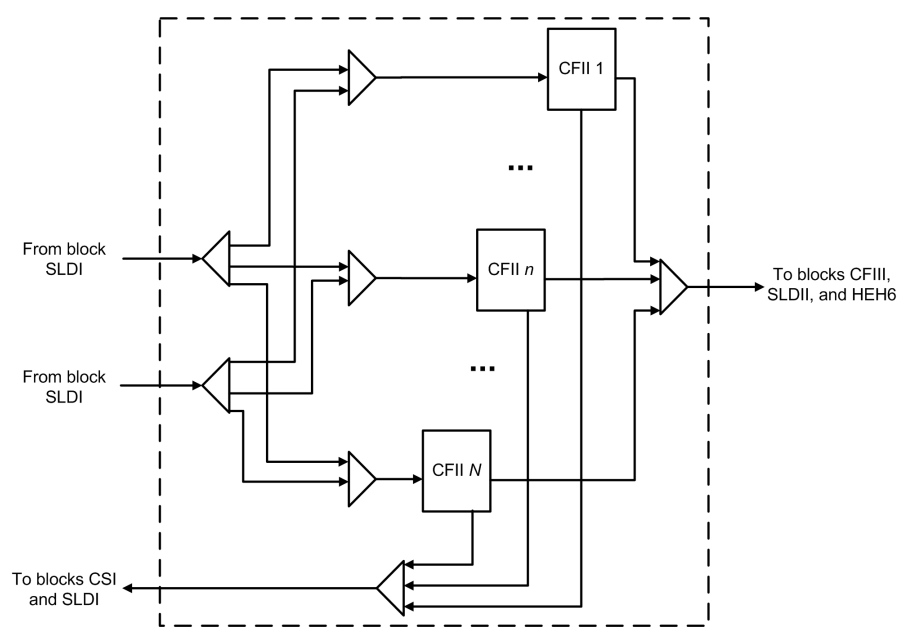

a) Detailed model.

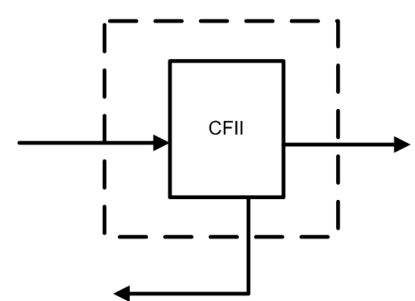

b) Aggregated model.

Figure 10 


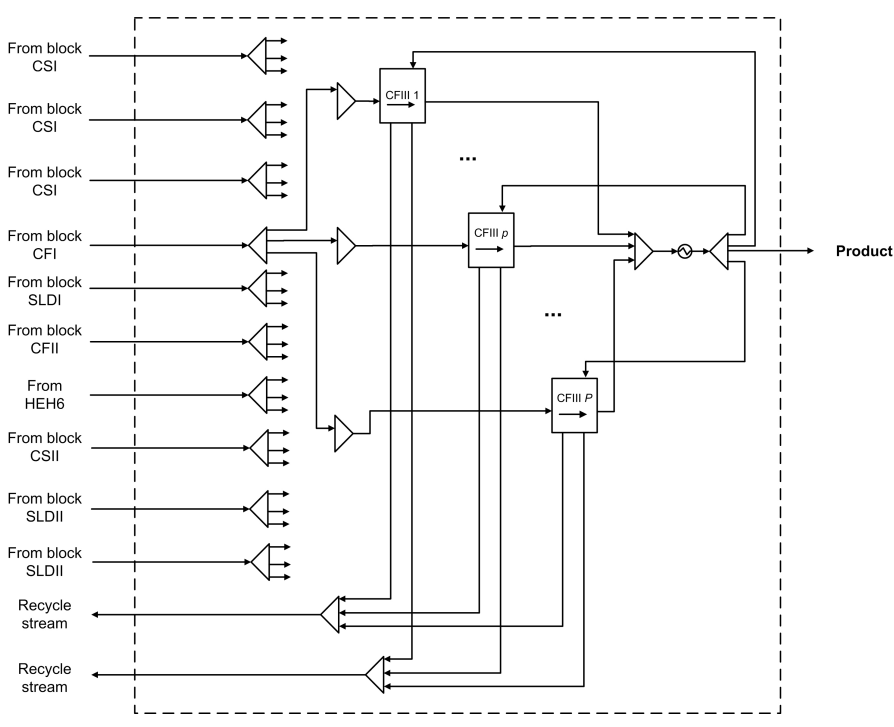

a) Detailed model.

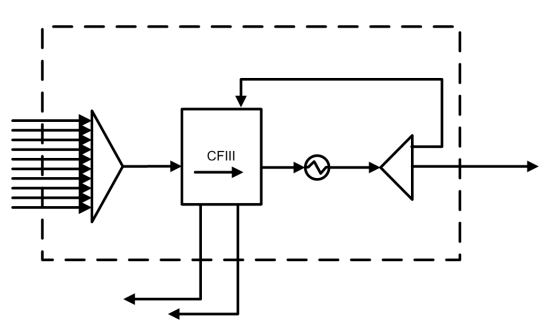

b) Aggregated model.

Figure 11 


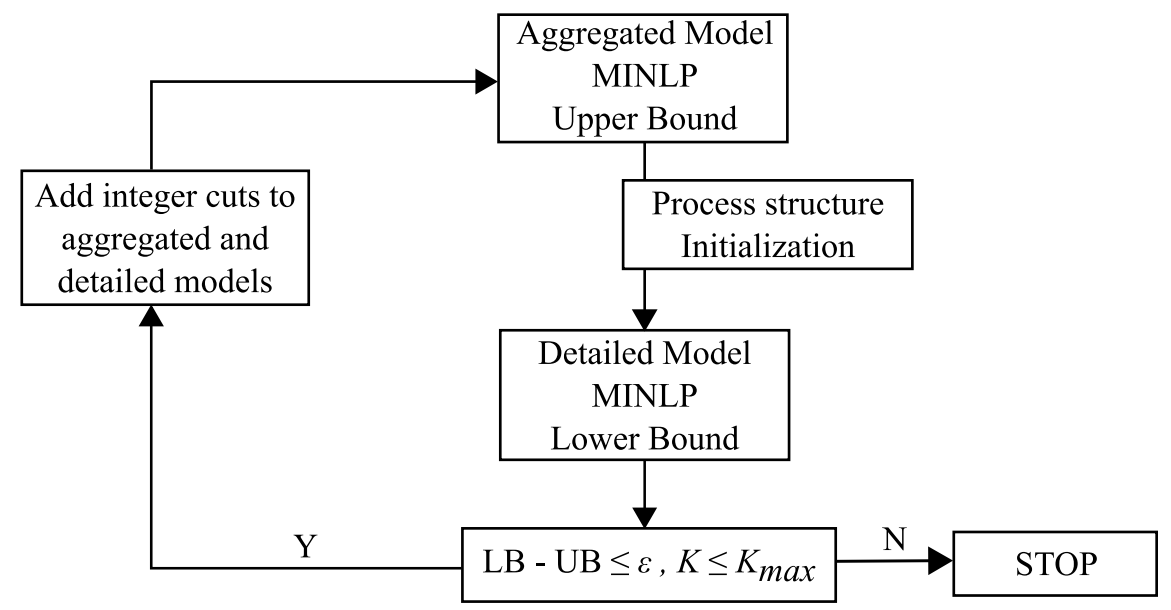

Figure 12 


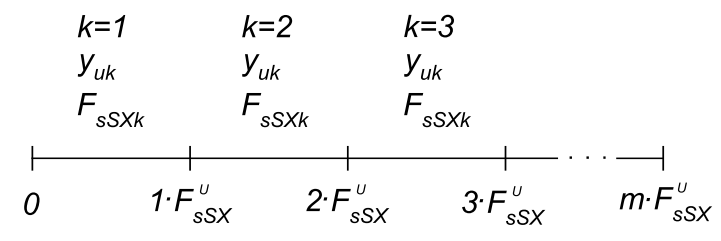

Figure 13 


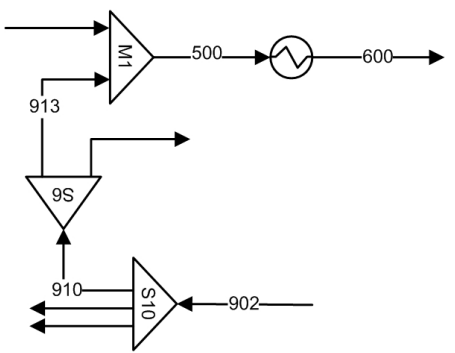

a) Extract from the aggregated model.

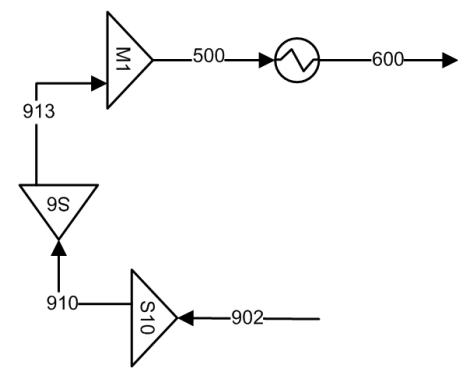

b) The streams with zero flowrate (streams with no label on the left figure) in the solution of the aggregated model are removed.

Figure 14

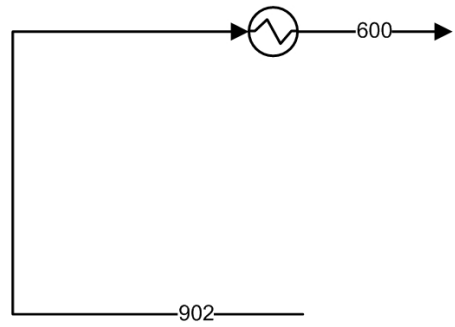

c) Mixers with one input stream and splitters with one output stream are removed from the superstructure of the detailed model. 


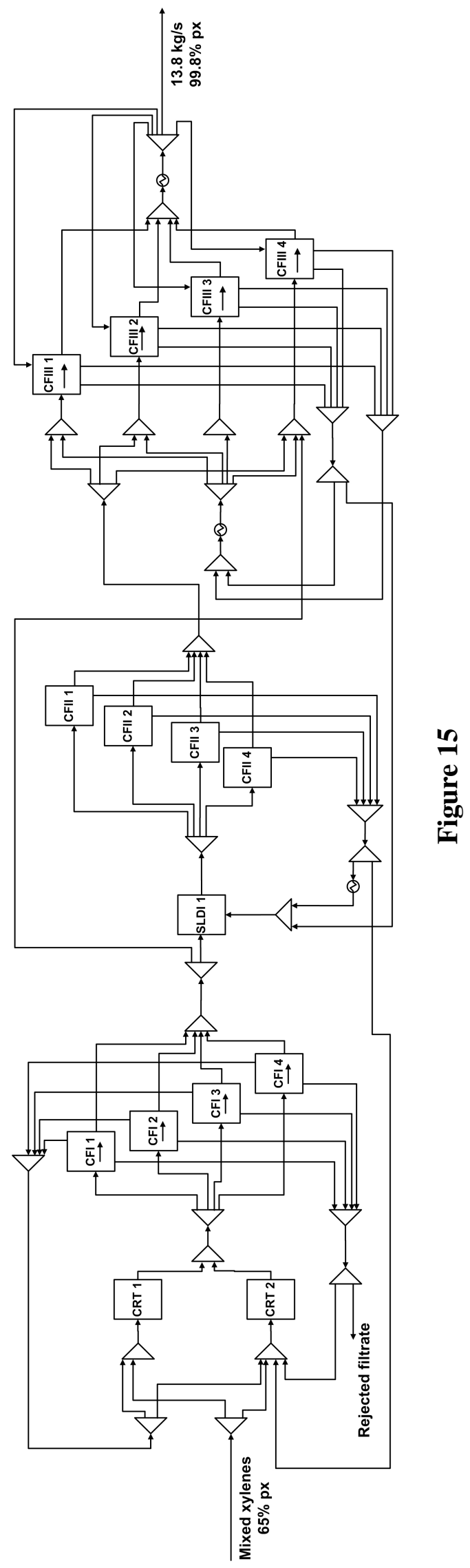




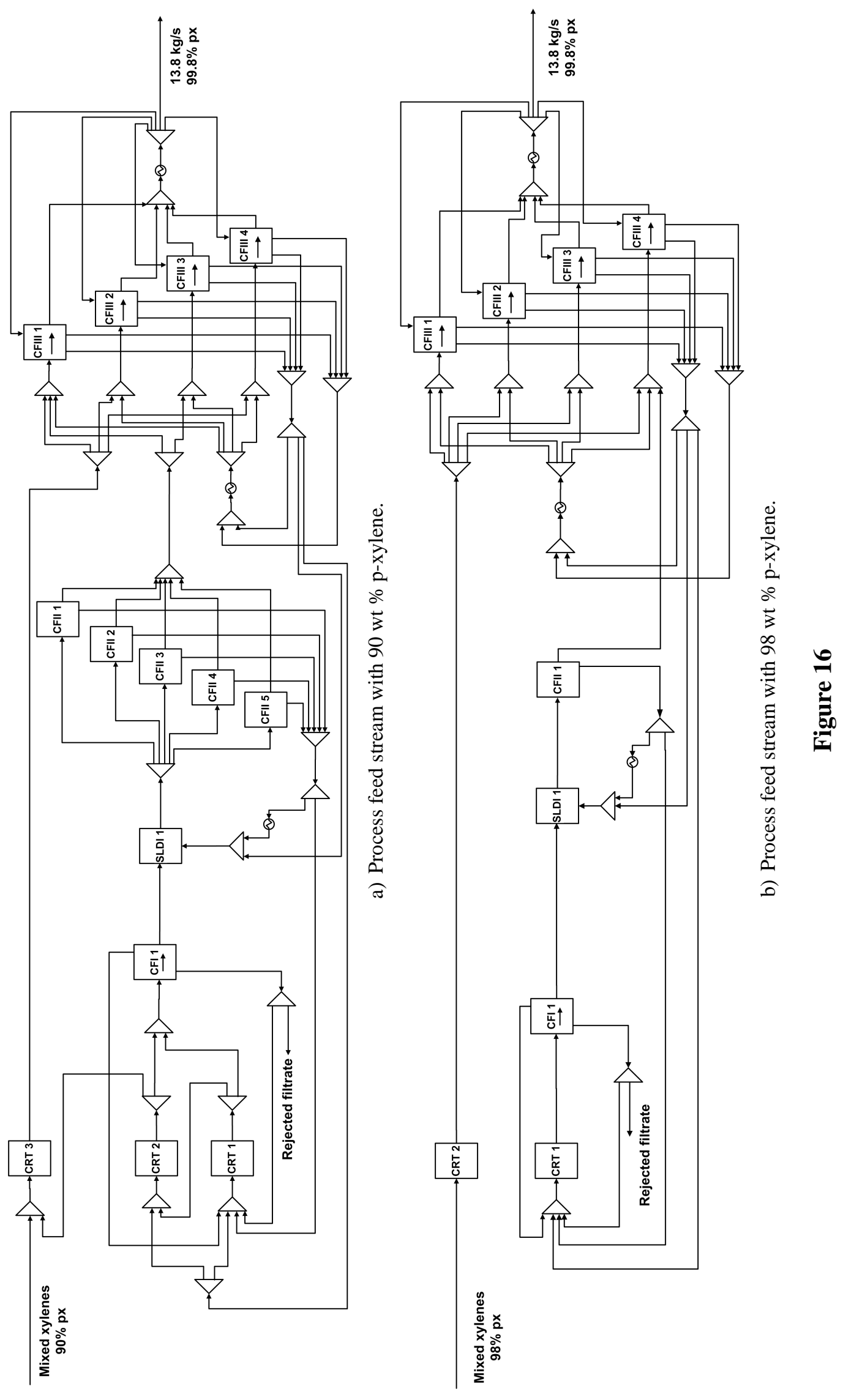




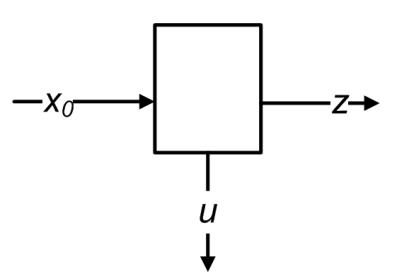

a)

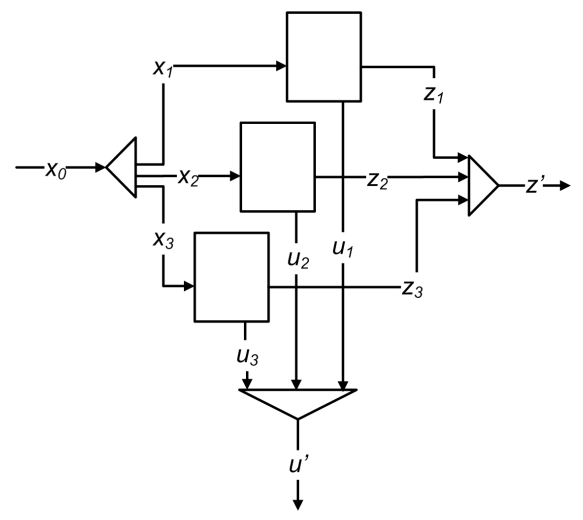

b)

Figure 17 


\section{List of Tables}

Table 1 Boiling and freezing point of each component in the process feed

Table 2 Size of each model for decomposition and simultaneous solution for Case III

Table 3 Best solutions, in terms of objective function, feed flowrate and number of units in each block, obtained with two different initialization approaches

Table 4 Results at each iteration level for Case I

Table 5 Results at each iteration level for Case II

Table 6 Results at each iteration level for Case III

Table 7 Optimum results for the total annual cost, investment and operating percentage costs for each case 
Table 1

\begin{tabular}{lcccc}
\hline \multirow{2}{*}{ Boiling point $(\mathrm{C}, 1$ bar $)$} & 144.4 & 139.1 & 138.4 & 136.2 \\
Freezing point $(\mathrm{C})$ & -25.2 & -47.9 & 13.3 & -95.0 \\
\hline
\end{tabular}


Table 2

\begin{tabular}{|c|c|c|c|c|c|}
\hline \multirow[t]{2}{*}{ Model } & \multirow[t]{2}{*}{ 0-1 Variables } & \multirow{2}{*}{$\begin{array}{c}\text { Continuous } \\
\text { variables }\end{array}$} & \multirow[t]{2}{*}{ Equations } & \multicolumn{2}{|c|}{ Items eliminated ${ }^{*}$} \\
\hline & & & & Streams & Splitters \\
\hline Aggregated & 53 & 1096 & 1177 & & \\
\hline Detailed $^{\dagger}$ & 79 & 1646 & 1786 & 162 & 32 \\
\hline Detailed $^{\ddagger}$ & 102 & 2838 & 2810 & & \\
\hline
\end{tabular}

$\uparrow$ - Two-level decomposition. $\ddagger$ - Initial superstructure, ${ }^{*}$ - Items removed in Step 5 
Table 3

\begin{tabular}{|c|c|c|c|c|c|c|c|}
\hline & \multirow{2}{*}{$\begin{array}{c}Z^{*} \\
\text { (m.u./year) }\end{array}$} & \multirow{2}{*}{$\begin{array}{l}\text { Feed flowrate } \\
\qquad(\mathrm{kg} / \mathrm{s})\end{array}$} & \multicolumn{5}{|c|}{ Number of units } \\
\hline & & & CRT & CFI & CFII & SLD & CFIII \\
\hline Case $\mathrm{I}^{\dagger}$ & 101.2 & 23.6 & 2 & 4 & 4 & 1 & 4 \\
\hline Case $I^{\ddagger}$ & 101.4 & 23.6 & 3 & 4 & 3 & 1 & 4 \\
\hline Case $\mathrm{II}^{\dagger}$ & 99.5 & 15.8 & 3 & 1 & 5 & 1 & 4 \\
\hline Case $\mathrm{II}^{\ddagger}$ & 100.3 & 15.8 & 5 & 1 & 3 & 1 & 4 \\
\hline
\end{tabular}


Table 4

\begin{tabular}{|c|c|c|c|c|c|c|c|}
\hline \multirow[b]{2}{*}{$K$} & \multirow[b]{2}{*}{$Z_{U}^{K}$} & \multirow[b]{2}{*}{$\begin{array}{c}Z_{L}^{K \dagger} \\
\text { (m.u./year) }\end{array}$} & \multirow[b]{2}{*}{$Z_{L}^{K \ddagger}$} & \multicolumn{2}{|c|}{ Aggregated } & \multicolumn{2}{|c|}{ Detailed } \\
\hline & & & & $\begin{array}{c}\mathrm{SBB}^{* *} \\
(\mathrm{CPU} \mathrm{s})\end{array}$ & $\begin{array}{r}\text { DICOPT } \\
(\mathrm{CPU} s)\end{array}$ & $\begin{array}{c}\mathrm{SBB}^{* *} \\
(\mathrm{CPU} \mathrm{s})\end{array}$ & $\begin{array}{r}\text { DICOPT } \\
\text { (CPU s) }\end{array}$ \\
\hline 1 & 105.1 & 101.5 & 102.3 & 219.4 & 3.9 & 613.2 & 10.7 \\
\hline 2 & 104.1 & 104.1 & 104.8 & 47.7 & 3.5 & 1760.4 & 4.9 \\
\hline 3 & 104.2 & 101.3 & 105.2 & 68.7 & 2.1 & 75.7 & 22.2 \\
\hline 4 & 104.4 & $101.2^{*}$ & 101.4 & 28.0 & 1.3 & 881.0 & 5.3 \\
\hline 6 & 104.3 & - & - & - & 12.6 & - & - \\
\hline 7 & 104.7 & 102.7 & - & 52.0 & 7.8 & 603.3 & 11.5 \\
\hline 8 & 104.4 & - & - & 130.6 & 2.4 & - & - \\
\hline 9 & 104.6 & 105.8 & 106.1 & 63.9 & 1.8 & 109.1 & 6.3 \\
\hline
\end{tabular}

$\dagger^{\dagger}$ - Updated $Z_{L}^{K}$ in Step $8 .{ }^{\ddagger}$ - $Z_{L}^{K}$ yielded by the detailed model before check step 8 .

* - Optimal solution. ${ }^{* *}$ - Maximum number of nodes set to 500. 
Table 5

\begin{tabular}{|c|c|c|c|c|c|c|c|}
\hline \multirow[b]{2}{*}{$K$} & \multirow[b]{2}{*}{$Z_{U}^{K}$} & \multirow[b]{2}{*}{$\begin{array}{c}Z_{L}^{K \dagger} \\
\text { (m.u./year) }\end{array}$} & \multirow[b]{2}{*}{$Z_{L}^{K \ddagger}$} & \multicolumn{2}{|c|}{ Aggregated } & \multicolumn{2}{|c|}{ Detailed } \\
\hline & & & & $\begin{array}{c}\mathrm{SBB}^{* *} \\
(\mathrm{CPU} \mathrm{s})\end{array}$ & $\begin{array}{r}\text { DICOPT } \\
\text { (CPU s) }\end{array}$ & $\begin{array}{c}\mathrm{SBB}^{* *} \\
(\mathrm{CPU} \mathrm{s})\end{array}$ & $\begin{array}{r}\text { DICOPT } \\
\text { (CPU s) }\end{array}$ \\
\hline 1 & 102.7 & 100.2 & - & 68.7 & 6.3 & 956.53 & 7.9 \\
\hline 2 & 101.9 & $99.5^{*}$ & - & 38.8 & 8.3 & 786.58 & 18.2 \\
\hline 3 & 102.1 & 102.9 & 104.4 & 16.2 & 3.1 & - & 82.1 \\
\hline
\end{tabular}

$\dagger^{\dagger}$ - Updated $Z_{L}^{K}$ in Step $8 .{ }^{\ddagger}-Z_{L}^{K}$ yielded by the detailed model before check step 8 .

* - Optimal solution. ** - Maximum number of nodes set to 500 . 
Table 6

\begin{tabular}{|c|c|c|c|c|c|c|c|}
\hline \multirow[b]{2}{*}{$K$} & \multirow[b]{2}{*}{$Z_{U}^{K}$} & \multirow[b]{2}{*}{$\begin{array}{c}Z_{L}^{K \dagger} \\
\text { (m.u./year) }\end{array}$} & \multirow[b]{2}{*}{$Z_{L}^{K \ddagger}$} & \multicolumn{2}{|c|}{ Aggregated } & \multicolumn{2}{|c|}{ Detailed } \\
\hline & & & & $\begin{array}{c}\mathrm{SBB}^{* *} \\
(\mathrm{CPU} \mathrm{s})\end{array}$ & $\begin{array}{r}\text { DICOPT } \\
\text { (CPU s) }\end{array}$ & $\begin{array}{c}\mathrm{SBB}^{* *} \\
(\mathrm{CPU} \mathrm{s})\end{array}$ & $\begin{array}{r}\text { DICOPT } \\
\text { (CPU s) }\end{array}$ \\
\hline 1 & 98.7 & 100.5 & 100.7 & 23.8 & 3.1 & 362.87 & 13.3 \\
\hline 2 & 98.4 & $98.4^{*}$ & 99.3 & 28.2 & 2.9 & 32.12 & 5.5 \\
\hline
\end{tabular}

${ }^{\dagger}$ - Updated $Z_{L}^{K}$ in Step $8 .{ }^{\ddagger}-Z_{L}^{K}$ yielded by the detailed model before check step 8 .

* - Optimal solution. ** - Maximum number of nodes set to 100. 
Table 7

\begin{tabular}{cccc}
\hline Cases & Total cost (m.u./year) & Investment cost $(\%)$ & Operating cost (\%) \\
\hline I & 101.2 & 5.4 & 94.6 \\
II & 99.5 & 2.8 & 97.2 \\
III & 98.4 & 0.8 & 99.2 \\
\hline
\end{tabular}

